K. S. Thygesen ${ }^{1}$ and A. Rubio ${ }^{2}$

${ }^{1}$ Center for Atomic-scale Materials Design (CAMD), Department of Physics, Technical University of Denmark, 2850 Kgs. Lyngby, Denmark ${ }^{2}$ Nano Bio Spectroscopy Group and European Theoretical Spectroscopy Facility (ETSF). Departamento de Fisica de Materiales, Unidad de Materiales Centro Mixto CSIC-UPV/EHU Universidad del Pas Vasco, Edificio Korta, Avd. Tolosa 72, E-20018 Donostia, Spain. 


\title{
CORRELATED ELECTRON TRANSPORT IN MOLECULAR JUNCTIONS
}

\author{
K. S. Thygesen and A. Rubio
}

\section{INTRODUCTION}

The dimensions of conventional silicon based electronics devices will soon be so small that quantum effects, such as electron tunneling and energy quantization, will begin to influence and eventually limit the device functionality. On the other hand molecular electronics is based on the idea of constructing electronic devices from bottom up using organic molecules as basic building blocks and in this way integrate the quantum nature of the charge carriers directly in the design (Cuniberti et al. 2005 and Joachim et al. 2000).

Over the last decade it has become possible to capture individual nanostructures between metal contacts and measure the electrical properties of the resulting junction. The types of nanostructures vary all the way from a single hydrogen molecule (Smit et al. 2002) over organic molecules (Reichert 
et al. 2002) to metallic chains of single atoms (Yanson et al. 1998) to carbon nanotubes suspended over several nanometers (Nygard et al. 2000) and inorganic nanowires and biochromophores (Cuniberti et al. 2005). The physics of these systems is highly non-classical showing intriguing phenomena such as quantized conductance, conductance oscillations, strong electron-phonon coupling, Kondo physics, and Coulomb blockade. For this reason a microscopic, i.e. quantum mechanical, understanding of nanoscale systems out of equilibrium is fundamental for the future development of molecular electronics.

The theoretical description of electron transport in molecules (we often use the term molecule to cover a general nanostructure) represents a central challenge in computational nanoscience. In principle, the problem involves an open quantum system of electrons interacting with each other and the surrounding nuclei under the influence of an external bias voltage. Fortunately, due to the large difference in mass between electrons and nuclei, it is often a good approximation to regard the nuclei as classical charges fixed in their equilibrium positions - at least for sufficiently low temperature and bias voltage. This reduces the problem to interacting electrons moving through the static potential created by the frozen lattice of nuclei.

Further simplification is obtained by replacing the electron-electron interactions by a meanfield potential like is done in Hartree-Fock (HF) and Kohn-Sham (KS) theory. Within such independent-particle approximations Landauer's formula (Landauer 1970) applies, giving the conductance as the (elastic) transmission probability for electrons at the Fermi level times the conductance unit $G_{0}=2 e^{2} / h$. Landauer's formula and, in particular, its equivalent formulation in terms of nonequilibrium Green's functions (NEGF) (Meir and Wingreen 1992), has formed the basis for almost all calculations of quantum transport in nano-scale systems. First-principles calculations are usually based on the KS scheme of Density Functional Theory (DFT) with a local exchange-correlation functional (Taylor et al. 2001, Brandbyge et al. 2002). These DFT transport schemes have been successfully applied to systems characterized by strong coupling between the molecule and the electrodes (Thygesen and Jacobsen 2005, Garci-Suarez et al. 2005), but systematically overestimates the conductance of weakly coupled systems (Heurich et al. 2002, Quek et al. 2007). Recently, it has been shown that the use of self-interaction corrected exchange-correlation functionals improves the agreement with experiments for such systems (Toher et al. 2005). However, such functionals contain parameters which basically controls the size of the energy gap between highest occupied (HOMO) and lowest unoccupied (LUMO) molecular orbitals which questions the predictive power of such an approach. 
Apart from the problems related to the position of molecular energy levels, there are a number of electronic effects originating from the two-body nature of the electron-electron interaction, which cannot - even in principle - be described within a single-particle picture. These include strong correlation effects like Kondo effects and Coulomb blockade (Goldhaber et al. 1998, Costi et al. 1994), renormalization of molecular levels by dynamic screening (Neaton et al. 2006, Kubatkin et al. 2003), and life-time reduction due to quasiparticle scattering (Thygesen 2008). As we shall see in this chapter, such dynamic correlation effects can have a dramatic influence on the electrical properties - in particular far from equilibrium.

In this chapter we describe how electronic correlation effects can be included in transport calculations using many-body perturbation theory within the Keldysh nonequilibrium Green's function formalism. Specifically, we use the so-called $G W$ self-energy method ( $G$ denotes the Green's function and $W$ is the screened interaction) which has been successfully applied to describe quasiparticle excitations in weakly correlated systems (Hybertsen and Louie 1986, Onida et al. 2002). To make the problem tractable, we limit the $G W$ description to a central region containing the nanostructure of interest and part of the leads, while the (rest of the) metallic leads are treated at a meanfield level. The rationale behind this division is that the transport properties to a large extent are determined by the narrowest part of the conductor, i.e. the molecule, while the leads mainly serve as particle reservoirs (a proper inclusion of substrate polarization effects require that a sufficiently large part of the leads are included in the central region).

The use of nonequilibrium many-body perturbation theory is only one out of several methods to include correlation effects in quantum transport. In another approach the density matrix is obtained from a many-body wave function and the non-equilibrium boundary conditions are invoked by fixing the occupation numbers of left- and right going states (Delaney and Greer 2004). Exact diagonalization within the molecular subspace has been combined with rate equations to calculate tunneling currents to first order in the lead-molecule coupling strength (Hettler et al. 2003). The linear response conductance of jellium quantum point contacts has been addressed on the basis of the Kubo formula which in principle allows correlation effects to be incorporated through the response function (Bokes et al. 2007). The time-dependent version of density functional theory has also been used as framework for quantum transport (Stefanucci et al. 2007, Di Ventra and Todorov 2004). This scheme is particularly useful for simulating transients and high frequency ac-responses and can in principle include correlations via non-adiabatic exchange-correlation Kernels.

In section 2 we formulate the quantum transport problem and give a brief 
introduction to the nonequilibrium Green's function formalism. In section 3 we present the nonequilibrium $G W$ equations and discuss the important concept of conserving approximations. In section 4.1 we obtain an expression for the current within the NEGF formalism which holds for interactions in the central region. It is demonstrated, both analytically and by numerical examples, that a self-consistent evaluation of the $G W$ self-energy is fundamentally important for nonequilibrium transport as it - in contrast to the popular non self-consistent approach - ensures the validity of the continuity equation. In section 5, with the aim of identifying universal trends, we study a generic two-level model of a molecular junction. It is demonstrated how dynamic polarization effects renormalize the molecular levels, and a physical interpretation in terms of constrained total energy differences is provided. The application of a bias voltage is shown to enhance the dynamic polarization effects. Moreover, quasiparticle scattering becomes increasingly important at larger bias leading to a significant broadening of the molecular resonances. These effects, which are all beyond the single-particle approximation, have large impact on the calculated $I V$ curve. In section 6 we combine the $G W$ transport scheme with DFT (for the leads) and a Wannier function basis set, and apply it to two prototypical junctions, namely a benzene molecule coupled to featureless leads and a hydrogen molecule between infinite Pt chains, and the results are analyzed using the knowledge obtained from the twolevel model. It is found that non self-consistent $G_{0} W_{0}$ calculations depend crucially on the $G_{0}$ (whether it is the Hartree-Fock or Kohn-Sham Green's function). This together with its non conserving nature suggests that $G W$ transport calculations should be performed self-consistently.

This chapter is a summary of recent work by the authors on incorporating many-body correlation effects in quantum transport, see Thygesen and Rubio 2007, Thygesen and Rubio 2008, and Thygesen 2008.

\section{FORMALISM}

In this section we formulate the quantum transport problem and review the elements of the Keldysh Green's function theory needed for its solution. For more detailed introductions to the subject we refer to the books by Leeuwen et al. 2006 and Haug and Jauho 1998. To limit the technical details we specialize to the case of an orthogonal basis set and refer to Thygesen (2006) for a generalization to the non-orthogonal case. 


\subsection{Model}

We consider a quantum conductor consisting of a central region $(C)$ connected to left $(L)$ and right $(R)$ leads. For times $t<t_{0}$ the three regions are decoupled from each other, each being in thermal equilibrium with a common temperature and chemical potentials $\mu_{L}, \mu_{C}$, and $\mu_{R}$, respectively (see Fig. 1). At $t=t_{0}$ the coupling between the three subsystems is switched on and a current starts to flow as the electrode with higher chemical potential discharges through the central region into the lead with lower chemical potential. Our aim is to calculate the steady state current which arise after the transient has died out. Notice that the duration of the steady state is determined by the size of the leads which we henceforth take to be infinite.

The single-particle state space of the electrons, $\mathcal{H}$, is spanned by the orthonormal basis set $\left\{\phi_{i}\right\}$. The orbitals $\phi_{i}$ are assumed to be localized such that $\mathcal{H}$ can be decomposed into a sum of orthogonal subspaces corresponding to the division of the system into leads and central region, i.e. $\mathcal{H}=\mathcal{H}_{L}+$ $\mathcal{H}_{C}+\mathcal{H}_{R}$. We will use the notation $i \in \alpha$ to indicate that $\phi_{i} \in \mathcal{H}_{\alpha}$ for some $\alpha \in\{L, C, R\}$.

The non-interacting part of the Hamiltonian of the connected system is written

$$
\hat{h}=\sum_{\substack{i, j \in \in \\ L, C, R}} \sum_{\sigma=\uparrow \downarrow} h_{i j} c_{i \sigma}^{\dagger} c_{j \sigma}
$$

where $i, j$ run over all basis states of the system. For $\alpha, \beta \in\{L, C, R\}$, the operator $\hat{h}_{\alpha \beta}$ is obtained by restricting $i$ to region $\alpha$ and $j$ to region $\beta$ in Eq. (1). Occasionally we shall write $\hat{h}_{\alpha}$ instead of $\hat{h}_{\alpha \alpha}$. We assume that there is no direct coupling between the two leads, i.e. $\hat{h}_{L R}=\hat{h}_{R L}=0$ (this condition can always be fulfilled by increasing the size of the central region since the basis functions are localized). We introduce a special notation for the "diagonal" of $\hat{h}$,

$$
\hat{h}_{0}=\hat{h}_{L L}+\hat{h}_{C C}+\hat{h}_{R R} .
$$

It is instructive to note that $\hat{h}_{0}$ does not describe the three regions in physical isolation from each other, but rather the contacted system without interregion hopping. We allow for interactions between electrons inside the central region. The most general form of such a two-body interaction is,

$$
\hat{V}=\sum_{\substack{i j k l \in C \\ \sigma \sigma^{\prime}}} V_{i j, k l} c_{i \sigma}^{\dagger} c_{j \sigma^{\prime}}^{\dagger} c_{l \sigma^{\prime}} c_{k \sigma} .
$$

The full Hamiltonian describing the system at time $t$ can then be written

$$
\hat{H}(t)= \begin{cases}\hat{H}_{0}=\hat{h}_{0}+\hat{V} & \text { for } t<t_{0} \\ \hat{H}=\hat{h}+\hat{V} & \text { for } t>t_{0}\end{cases}
$$


Notice, that we use small letters for non-interacting quantities while the subscript 0 refers to uncoupled quantities. At this point we shall not be concerned about the actual value of the matrix elements $h_{i j}$ and $V_{i j, k l}$ as this is unimportant for the general formalism discussed here.

For times $t<t_{0}$ each of the three subsystems are assumed to be in thermal equilibrium characterized by their equilibrium density matrices. For the left lead we have

$$
\hat{\varrho}_{L}=\frac{1}{Z_{L}} \exp \left(-\beta\left(\hat{h}_{L}-\mu_{L} \hat{N}_{L}\right)\right)
$$

with

$$
Z_{L}=\operatorname{Tr}\left[\exp \left(-\beta\left(\hat{h}_{L}-\mu_{L} \hat{N}_{L}\right)\right)\right] .
$$

Here $\beta$ is the inverse temperature and $\hat{N}_{L}=\sum_{\sigma, i \in L} c_{i \sigma}^{\dagger} c_{i \sigma}$ is the number operator of lead $L$. $\hat{\varrho}_{R}$ and $Z_{R}$ are obtained by replacing $L$ by $R$. For $\hat{\varrho}_{C}$ and $Z_{C}$ we must add $\hat{V}$ to the Hamiltonian in the exponential to account for the correlations. The initial state of the whole system is given by

$$
\hat{\varrho}=\hat{\varrho}_{L} \hat{\varrho}_{C} \hat{\varrho}_{R} \text {. }
$$

If $\hat{V}$ is not included in $\hat{\varrho}_{C}$ we obtain the uncorrelated, or non-interacting, initial state $\hat{\varrho}_{n i}$. Because $\hat{H}_{0}\left(\hat{h}_{0}\right)$ describes the contacted system in the absence of inter-region hopping, $\hat{\varrho}\left(\hat{\varrho}_{n i}\right)$ do not describe the three regions in physical isolation. In other words the three regions are only decoupled at the dynamic level for times $t<t_{0}$.

\subsection{Contour-ordered Green's function}

To treat nonequilibrium problems it is useful to extend the time-propagation operator from the real time axis to the so-called Keldysh contour, $\mathcal{C}_{I}$, depicted in Fig. 2(a). The contour is defined in the complex plane and runs along the real axis from $t_{0}$ to infinity, then back to $t_{0}$ and vertically down to $t_{0}-i \beta$. When $\tau$ and $\tau^{\prime}$ denote points on the Keldysh contour, the generalized timepropagation operator is defined by

$$
\hat{U}\left(\tau^{\prime}, \tau\right)=T e^{-i \int_{\tau}^{\tau^{\prime}} \mathrm{d} \bar{\tau} \hat{H}(\bar{\tau})}
$$

where $T$ orders a product of operators according to their time argument on the contour (late goes left). The integral is taken along $\mathcal{C}_{I}$. So far we have defined the Hamiltonian $\hat{H}(\tau)$ only for $\tau$ on the real axis. For Eq. (8) to make sense we must extend $\hat{H}(\tau)$ to the vertical branch of $\mathcal{C}_{I}$ which we will do in a moment. The contour-ordered single-particle GF relevant for our transport problem is defined by

$$
G_{i \sigma, j \sigma^{\prime}}\left(\tau, \tau^{\prime}\right)=-i \operatorname{Tr}\left\{\hat{\varrho} T\left[c_{H, i \sigma}(\tau) c_{H, j \sigma^{\prime}}^{\dagger}\left(\tau^{\prime}\right)\right]\right\},
$$



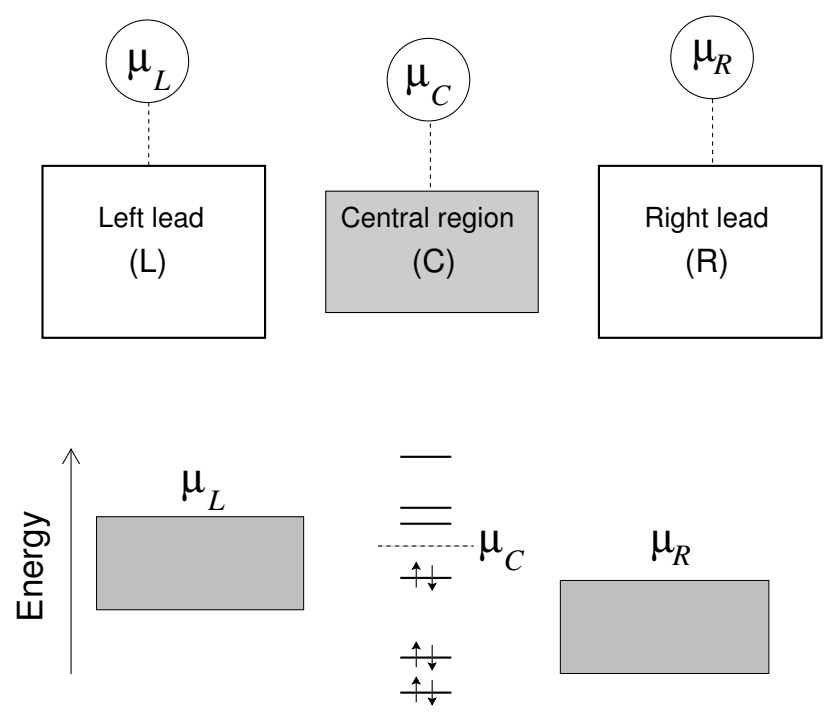

Figure 1: Before the coupling between the leads and central region is established, the three subsystems are in equilibrium with chemical potentials $\mu_{L}, \mu_{T}$, and $\mu_{C}$, respectively. Reprinted with permission from Thygesen and Rubio, Phys. Rev. B 77, 115333 (2008). Copyright 2008 by the American Physical Society.

where e.g. $c_{H, i \sigma}(\tau)=\hat{U}\left(t_{0}, \tau\right) c_{i \sigma} \hat{U}\left(\tau, t_{0}\right)$ and $\hat{\varrho}$ is the state of the system at time $t_{0}$. Notice that when evaluating $c_{H, i \sigma}(\tau)$ for $\tau$ on the real axis it does not matter whether $\tau$ is chosen on the upper or lower part of the contour. Notice also that when $\tau$ and $\tau^{\prime}$ are both in the vertical branch, $G$ is nothing but the Matsubara GF known from the equilibrium theory. For later reference we also note that a non-equilibrium GF is completely defined once (i) the Hamiltonian governing the dynamics and (ii) the initial state have been specified. Since the Hamiltonian contains no spin-flip processes the GF is diagonal in spin space, i.e. $G_{i \sigma, j \sigma^{\prime}}=\delta_{\sigma \sigma^{\prime}} G_{i j}$ and we therefore suppress the spin-dependence in the following.

If we define $\hat{H}(\tau)$ along the vertical part of the contour to be

$$
\hat{H}(\tau)=\sum_{\alpha=L, C, R}\left(\hat{h}_{\alpha}-\mu_{\alpha} \hat{N}_{\alpha}\right)+\hat{V}
$$

we see that $\hat{U}\left(t_{0}-i \beta, t_{0}\right)=Z \hat{\varrho}$. We use this observation to write

$$
G_{i j}\left(\tau, \tau^{\prime}\right)=-i \frac{\operatorname{Tr}\left\{T\left[e^{-i \int_{\mathcal{C}_{I}} \mathrm{~d} \bar{\tau} \hat{H}(\bar{\tau})} c_{i}(\tau) c_{j}^{\dagger}\left(\tau^{\prime}\right)\right]\right\}}{\operatorname{Tr}\left\{T e^{-i \int_{\mathcal{C}_{I}} \mathrm{~d} \bar{\tau} \hat{H}(\bar{\tau})}\right\}} .
$$



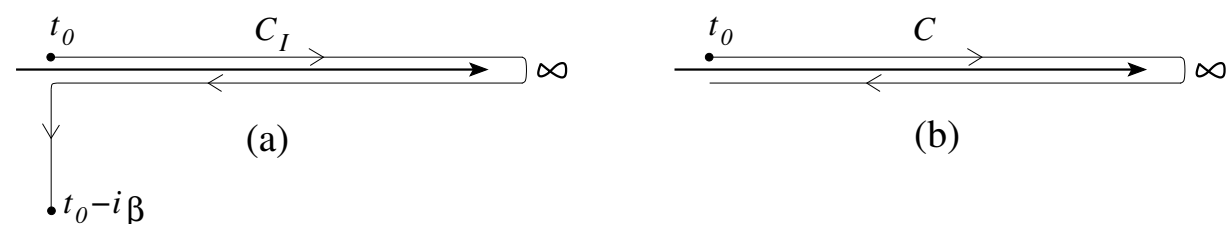

(b)

Figure 2: (a) The Keldysh contour $\mathcal{C}_{I}$. The dynamics of the system is governed by the Hamiltonian $\hat{H}(\tau)$ on the horizontal branch, while the initial state is defined by $\hat{H}(\tau)$ on the vertical branch. (b) The Keldysh contour $\mathcal{C}$, used when correlations in the initial state are neglected.

Here the time-argument of $c_{i}(\tau)$ and $c_{j}^{\dagger}\left(\tau^{\prime}\right)$ only serves to identify their position in the contour-ordering, i.e. they are not in the Heisenberg picture.

In order to obtain an expansion of $G_{i j}\left(\tau, \tau^{\prime}\right)$ in powers of $\hat{V}$, we switch to the interaction picture defined by the non-interacting Hamiltonian $\hat{h}(\tau)=$ $\hat{H}(\tau)-\hat{V}$. In this picture we have

$$
G_{i j}\left(\tau, \tau^{\prime}\right)=-i \frac{\operatorname{Tr}\left\{\hat{\varrho}_{n i} T\left[e^{-i \int_{\mathcal{C}_{I}} \mathrm{~d} \bar{\tau} \hat{V}_{h}(\bar{\tau})} c_{h, i}(\tau) c_{h, j}^{\dagger}\left(\tau^{\prime}\right)\right]\right\}}{\operatorname{Tr}\left\{\varrho_{n i} T e^{-i \int_{\mathcal{C}_{I}} \mathrm{~d} \bar{\tau} \hat{V}_{h}(\bar{\tau})}\right\}},
$$

where the time-dependence of the operators is governed by the evolution operator in Eq. (8) with $\hat{H}$ replaced by $\hat{h}$. The density matrix $\hat{\varrho}_{n i}$ is given by

$$
\hat{\varrho}_{n i}=\frac{\exp \left(-\beta \sum_{\alpha}\left(\hat{h}_{\alpha}-\mu_{\alpha} \hat{N}_{\alpha}\right)\right)}{\operatorname{Tr}\left\{\exp \left(-\beta \sum_{\alpha}\left(\hat{h}_{\alpha}-\mu_{\alpha} \hat{N}_{\alpha}\right)\right)\right\}},
$$

which differs from $\hat{\varrho}$ in that it does not contain interactions in the central region. From the identity

$$
Z \hat{\varrho}=Z_{0} \hat{\varrho}_{n i} T e^{-i \int_{t_{0}}^{t_{0}-i \beta} \mathrm{d} \bar{\tau} \hat{V}_{h}(\bar{\tau})}
$$

it is clear that the integration along the vertical branch of $\mathcal{C}_{I}$ in Eq. (12) accounts for the correlations in the initial state of region $C$. While it must be expected that the presence of initial correlations will influence the transient behavior of the current, it seems plausible that they will be washed out over time such that the steady state current will not depend on whether or not correlations are present in the initial state. In practice the neglect of initial correlations is a major simplification which allows us to work entirely on the real axis avoiding any reference to the imaginary time. For these reasons we shall neglect initial correlations in the rest of this paper. The GF can then 
be written

$$
G_{i j}\left(\tau, \tau^{\prime}\right)=-i \frac{\operatorname{Tr}\left\{\hat{\varrho}_{n i} T\left[e^{-i \int_{\mathcal{C}} \mathrm{d} \bar{\tau} \hat{V}_{h}(\bar{\tau})} c_{h, i}(\tau) c_{h, j}^{\dagger}\left(\tau^{\prime}\right)\right]\right\}}{\operatorname{Tr}\left\{\hat{\varrho}_{n i} T e^{-i \int_{\mathcal{C}} \mathrm{d} \bar{\tau} \hat{V}_{h}(\bar{\tau})}\right\}},
$$

where the contour $\mathcal{C}$ is depicted in Fig. 2(b). Eq. (15) is the starting point for a systematic series expansion of $G_{i j}$ in powers of $\hat{V}$. Since $\hat{\varrho}_{n i}$ is a mixed state of Slater determinants and the time-evolution is given by the non-interacting Hamiltonian, $\hat{h}$, Wick's theorem applies and leads to the standard Feynman rules with the exception that all time integrals are along the contour $\mathcal{C}$ and all Green's functions are contour-ordered. The Feynman diagrams should be constructed using the Green's function defined by $\hat{\varrho}_{n i}$ and $\hat{h}$,

$$
g_{i j}\left(\tau, \tau^{\prime}\right)=-i \operatorname{Tr}\left\{\hat{\varrho}_{n i} T\left[c_{h, i}(\tau) c_{h, j}^{\dagger}\left(\tau^{\prime}\right)\right]\right\}
$$

which describes the non-interacting electrons in the contacted system.

The diagrammatic expansion leads to the identification of a self-energy, $\Sigma$, as the sum of all irreducible diagrams with no external vertices. The GF is related to the self-energy and the non-interacting GF through Dyson's equation

$$
G\left(\tau, \tau^{\prime}\right)=g\left(\tau, \tau^{\prime}\right)+\int_{\mathcal{C}} \mathrm{d} \tau_{1} \mathrm{~d} \tau_{2} g\left(\tau, \tau_{1}\right) \Sigma\left(\tau_{1}, \tau_{2}\right) G\left(\tau_{2}, \tau^{\prime}\right),
$$

where matrix multiplication is implied. As we will see in Sec. 4.2, only the Green's function of the central region is needed for the calculation of the current, and we can therefore focus on the central-region submatrix of $G$. Since the interactions are limited to the central region, the self-energy matrix, $\Sigma_{i j}$, will be non-zero only when both $i, j \in C$, and it should therefore be safe to use the notation $\Sigma$ instead of $\Sigma_{C}$. Restricting Eq. (17) to the central region we have

$$
\begin{aligned}
G_{C}\left(\tau, \tau^{\prime}\right) & =g_{C}\left(\tau, \tau^{\prime}\right) \\
& +\int_{\mathcal{C}} \mathrm{d} \tau_{1} \mathrm{~d} \tau_{2} g_{C}\left(\tau, \tau_{1}\right) \Sigma\left(\tau_{1}, \tau_{2}\right) G_{C}\left(\tau_{2}, \tau^{\prime}\right)
\end{aligned}
$$

The free propagator, $g_{C}\left(\tau, \tau^{\prime}\right)$, is a non-equilibrium GF because $\hat{\varrho}_{n i}$ is not a stationary state of $\hat{h}$, i.e. $\left[\hat{\varrho}_{n i}, \hat{h}\right] \neq 0$. It is, however, not difficult to show that $g_{C}$ satisfies the following Dyson equation

$$
\begin{array}{r}
g_{C}\left(\tau, \tau^{\prime}\right)=g_{0, C}\left(\tau, \tau^{\prime}\right)+\int_{\mathcal{C}} \mathrm{d} \tau_{1} \mathrm{~d} \tau_{2} g_{0, C}\left(\tau, \tau_{1}\right) \\
{\left[\Sigma_{L}\left(\tau_{1}, \tau_{2}\right)+\Sigma_{R}\left(\tau_{1}, \tau_{2}\right)\right] g_{C}\left(\tau_{2}, \tau^{\prime}\right),}
\end{array}
$$


where $g_{0}$ is the equilibrium GF defined by $\hat{\varrho}_{n i}$ and $\hat{h}_{0}$. The coupling selfenergy due to lead $\alpha=L, R$ is given by

$$
\Sigma_{\alpha}\left(\tau, \tau^{\prime}\right)=h_{C \alpha} g_{0, \alpha}\left(\tau, \tau^{\prime}\right) h_{\alpha C}
$$

Notice the slight abuse of notation: $\Sigma_{\alpha}$ is not the $\alpha \alpha$ submatrix of $\Sigma$. In fact $\Sigma_{L}$ and $\Sigma_{R}$ are both matrices in the central region indices only. Combining Eqs. (18) and (19) we can write the Dyson equation for $G_{C}$,

$$
\begin{aligned}
G_{C}\left(\tau, \tau^{\prime}\right) & =g_{0, C}\left(\tau, \tau^{\prime}\right) \\
& +\int_{\mathcal{C}} \mathrm{d} \tau_{1} \mathrm{~d} \tau_{2} g_{0, C}\left(\tau, \tau_{1}\right) \Sigma_{t o t}\left(\tau_{1}, \tau_{2}\right) G_{C}\left(\tau_{2}, \tau^{\prime}\right),
\end{aligned}
$$

in terms of the equilibrium propagator of the non-interacting, uncoupled system, $g_{0}$, and the total self-energy

$$
\Sigma_{t o t}=\Sigma+\Sigma_{L}+\Sigma_{R}
$$

The total self-energy describes the combined effect of the interactions and the coupling to the leads.

\subsection{Real-time Green's functions}

In order to evaluate expectation values of single-particle observables we need the real-time correlation functions. We work with two correlation functions, also called the lesser and greater GFs, defined as

$$
\begin{aligned}
& G_{i j}^{<}\left(t, t^{\prime}\right)=i \operatorname{Tr}\left\{\hat{\varrho}_{n i} c_{H, j}^{\dagger}\left(t^{\prime}\right) c_{H, i}(t)\right\} \\
& G_{i j}^{>}\left(t, t^{\prime}\right)=-i \operatorname{Tr}\left\{\hat{\varrho}_{n i} c_{H, i}(t) c_{H, j}^{\dagger}\left(t^{\prime}\right)\right\} .
\end{aligned}
$$

Again, the use of $\hat{\varrho}_{n i}$ instead of $\hat{\varrho}$ amounts to neglecting initial correlations. Two other important real-time GFs are the retarded and advanced GFs defined by

$$
\begin{aligned}
& G_{i j}^{r}\left(t, t^{\prime}\right)=\theta\left(t-t^{\prime}\right)\left(G_{i j}^{>}\left(t, t^{\prime}\right)-G_{i j}^{<}\left(t, t^{\prime}\right)\right) \\
& G_{i j}^{a}\left(t, t^{\prime}\right)=\theta\left(t^{\prime}-t\right)\left(G_{i j}^{<}\left(t, t^{\prime}\right)-G_{i j}^{>}\left(t, t^{\prime}\right)\right) .
\end{aligned}
$$

The four GFs are related through

$$
G^{>}-G^{<}=G^{r}-G^{a} .
$$

The lesser and greater GFs are just special cases of the contour-ordered GF. For example $G^{<}\left(t, t^{\prime}\right)=G\left(\tau, \tau^{\prime}\right)$ when $\tau=t$ is on the upper branch of $\mathcal{C}$ 
and $\tau^{\prime}=t^{\prime}$ is on the lower branch. This can be used to derive a set of rules, sometimes referred to as the Langreth rules, for converting expressions involving contour-ordered quantities into equivalent expressions involving real-time quantities. We shall not list the conversion rules here, but refer to Haug and Jauho 1998 (no initial correlations) and Leeuwen et al. 2006 (including initial correlations). The usual procedure in non-equilibrium is then to derive the relevant equations on the contour using the standard diagrammatic techniques, and subsequently converting these equations to real time by means of the Langreth rules. An example of this procedure is given in Sec. 3.2 where the non-equilibrium $G W$ equations are derived.

\subsubsection{Equilibrium}

In equilibrium, the real-time GFs depend only on the time difference $t^{\prime}-t$. Fourier transforming with respect to this time difference then brings out the spectral properties of the system. In particular the spectral function

$$
A(\omega)=i\left[G^{r}(\omega)-G^{a}(\omega)\right]=i\left[G^{>}(\omega)-G^{<}(\omega)\right]
$$

shows peaks at the quasiparticle energies of the system, i.e. at the energies $E_{n}(N+1)-E_{m}(N)$ and $E_{m}(N)-E_{n}(N-1)$, where $n, m$ denote energy levels and $N$ the number of electrons. We thus see that the single-particle GF carries information about the electron addition- and removal energies. Clearly these are the types of excitations which are relevant in a transport situation where electrons are continuously added to and removed from the central region. In section 5.2 we discuss how many-body polarization effects renormalize the spectral function of a molecule adsorbed at a metal surface. In equilibrium we furthermore have the important fluctuation-dissipation theorem which relates the correlation functions to the spectral function and the Fermi-Dirac distribution function, $f$,

$$
\begin{aligned}
& G^{<}(\omega)=i f(\omega) A(\omega) \\
& G^{>}(\omega)=-i(1-f(\omega)) A(\omega) .
\end{aligned}
$$

The fluctuation-dissipation theorem follows from the Lehman representation which no longer holds out of equilibrium, and as a consequence one has to work explicitly with the correlation functions in non-equilibrium situations.

\subsubsection{Non-equilibrium steady state}

We shall make the plausible assumption that in steady state, all the real-time GFs depend only on the time-difference $t^{\prime}-t$. Moreover, we take the limit 
$t_{0} \rightarrow-\infty$. This will allow us to use the Fourier transform to turn convolutions in real time into products in frequency space. Applying the Langreth conversion rules to the Dyson equation (21), and Fourier transforming with respect to $t^{\prime}-t$ then leads to the following expression for the retarded GF of the central region

$$
G_{C}^{r}(\omega)=g_{0, C}^{r}(\omega)+g_{0, C}^{r}(\omega) \Sigma_{t o t}^{r}(\omega) G_{C}^{r}(\omega) .
$$

This equation can be inverted to yield the closed form

$$
G_{C}^{r}(\omega)=\left[(\omega+i \eta) I_{C}-h_{C}-\Sigma_{L}^{r}(\omega)-\Sigma_{R}^{r}(\omega)-\Sigma^{r}(\omega)\right]^{-1} .
$$

The equation for $G^{a}$ is obtained by replacing $r$ by $a$ and $\eta$ by $-\eta$ or, alternatively, from

$$
G_{C}^{a}(\omega)=\left[G_{C}^{r}(\omega)\right]^{\dagger}
$$

which follows from the assumption that the GFs depend on time differences only. For the correlation functions the conversion rules leads to the expression

$$
G_{C}^{</>}(\omega)=G_{C}^{r}(\omega) \Sigma_{t o t}^{</>}(\omega) G_{C}^{a}(\omega)+\Delta^{</>}(\omega)
$$

where

$$
\Delta^{</>}(\omega)=\left[I_{C}+G_{C}^{r}(\omega) \Sigma_{t o t}^{r}(\omega)\right] g_{0, C}^{</>}(\omega)\left[I_{C}+\Sigma_{t o t}^{a}(\omega) G_{C}^{a}(\omega)\right] .
$$

Using that $\Sigma_{\text {tot }}^{r / a}=\left(g_{0, C}^{r / a}\right)^{-1}-\left(G_{C}^{r / a}\right)^{-1}$ together with the equilibrium relations $g_{0, C}^{<}=-f\left(\omega-\mu_{C}\right)\left[g_{0, C}^{r}-g_{0, C}^{a}\right]$ and $g_{0, C}^{>}=-\left(f\left(\omega-\mu_{C}\right)-1\right)\left[g_{0, C}^{r}-g_{0, C}^{a}\right]$, we find that

$$
\begin{aligned}
\Delta^{<}(\omega) & =2 i \eta f\left(\omega-\mu_{C}\right) G_{C}^{r}(\omega) G_{C}^{a}(\omega) \\
\Delta^{>}(\omega) & =2 i \eta\left[f\left(\omega-\mu_{C}\right)-1\right] G_{C}^{r}(\omega) G_{C}^{a}(\omega) .
\end{aligned}
$$

If the product $G^{r} G^{a}$ is independent of $\eta$ we can conclude that $\Delta \rightarrow 0$ in the relevant limit of small $\eta$. It can in fact be shown that $\Delta$ always vanishes for interacting electrons while for non-interacting electrons $\Delta$ vanishes only when there are no bound states (Thygesen and Rubio 2008).

\subsubsection{Non-interacting electrons and lead self-energy}

In the special case of non-interacting electrons, the retarded and advanced GFs are independent of the initial state of the system, i.e. of the $\hat{\varrho}$ entering the GF. Moreover, if the dynamics is governed by a time-independent Hamiltonian, $g^{r}$ and $g^{a}$ depend only on the time difference $t^{\prime}-t$ (even if the initial state is not a stationary state). In this case the Fourier transform of 
the retarded and advanced GFs with respect to $t^{\prime}-t$ equals the resolvent of the Hamiltonian matrix $h$,

$$
g^{r / a}(\omega)=[(\omega \pm i \eta) I-h]^{-1},
$$

where $I$ is the identity matrix and $\eta$ is a positive infinitesimal. In our transport problem, the block-diagonal structure of $h_{0}$ allows us to obtain the non-interacting GF of the uncoupled system by inverting each block separately,

$$
g_{0, \alpha}^{r / a}(\omega)=\left[(\omega \pm i \eta) I-h_{\alpha}\right]^{-1}
$$

for $\alpha \in\{L, C, R\}$. Now it is in fact easy to show that the central region component of $g^{r / a}$ satisfies

$$
g_{C}^{r / a}(\omega)=\left[(\omega \pm i \eta) I-h_{C}-\Sigma_{L}^{r / a}(\omega)-\Sigma_{R}^{r / a}(\omega)\right]^{-1},
$$

with the retarded/advanced coupling self-energies given by

$$
\Sigma_{\alpha}^{r / a}(\omega)=h_{C \alpha} g_{0, \alpha}^{r / a}(\omega) h_{\alpha C} .
$$

Eqs. (40) and (41) give the retarded and advanced components of Eqs. (19) and (20), respectively. Notice that $\Sigma_{\alpha}^{r / a}$ depends on the applied bias voltage through $g_{0, \alpha}$ because the self-consistent field in the leads follow the chemical potential to ensure charge neutrality as sketched in the lower panel of Fig. 1. Assuming a symmetrically applied bias $\left(\mu_{L / R}=\varepsilon_{F} \pm V / 2\right)$ we have

$$
\Sigma_{L}^{r / a}(V ; \omega)=\Sigma_{L}^{r / a}(0 ; \omega-V / 2),
$$

with a similar relation for $\Sigma_{R}$.

Since $g_{0}$ is an equilibrium GF its lesser and greater components, and thus also $\Sigma_{\alpha}^{</>}$, follow from the fluctuation-dissipation theorem. In contrast, the correlation functions derived from $g$, which is a non-equilibrium function, must be calculated using the Keldysh equation (34).

\section{MANY-BODY SELF-ENERGY}

In this section we discuss two specific approximations to the many-body self-energy $\Sigma$ introduced in Eq. (17), namely the $G W$ and second Born (2B) approximations. Strictly speaking the $\Sigma$ of Eq. (17) contains the full effect of the interactions whereas the $G W$ and $2 \mathrm{~B}$ self-energies only describe exchange and correlation, i.e. they do not include the Hartree potential. The $G W$ selfenergy is obtained by summing an infinite set of Feynman diagrams - one 
diagram at each order of the interaction - while the $2 \mathrm{~B}$ approximation is exact to second order in the interaction (if performed self-consistently). In section 3.1 we introduce an effective interaction which leads to a particularly simple form of the self-energy equations and at the same time provides a means for reducing self-interaction errors in higher order diagrammatic expansions. In sections 3.2 and 3.3 we discuss the nonequilibrium $G W$ and $2 \mathrm{~B}$ equations using the effective interaction.

\subsection{Effective interaction}

The direct use of the full interaction Eq. (3) in a diagrammatic expansion is problematic as it introduces frequency dependent, four-index quantities, which quickly becomes difficult to store and handle numerically. For this reason we consider instead the effective interaction defined by

$$
\hat{V}_{\mathrm{eff}}=\sum_{i j, \sigma \sigma^{\prime}} \tilde{V}_{i \sigma, j \sigma^{\prime}} c_{i \sigma}^{\dagger} c_{j \sigma^{\prime}}^{\dagger} c_{j \sigma^{\prime}} c_{i \sigma}
$$

where

$$
\tilde{V}_{i \sigma, j \sigma^{\prime}}=V_{i j, i j}-\delta_{\sigma \sigma^{\prime}} V_{i j, j i}
$$

This expression follows by restricting the sum in the full interaction Eq. (3) to terms of the form $V_{i j, i j} c_{i \sigma}^{\dagger} c_{j \sigma^{\prime}}^{\dagger} c_{j \sigma^{\prime}} c_{i \sigma}$ and $V_{i j, j i} c_{i \sigma}^{\dagger} c_{j \sigma}^{\dagger} c_{j \sigma} c_{i \sigma}$.

The effective interaction is local in orbital space, i.e. it is a two-point function instead of a four-point function and thus resembles the real-space representation. Note, however, that in contrast to the real-space representation $\tilde{V}_{i \sigma, j \sigma^{\prime}}$ is spin-dependent. In particular the self-interactions, $\tilde{V}_{i \sigma, i \sigma}$, are zero by construction and consequently self-interaction (in the orbital basis) is avoided to all orders in a perturbation expansion in powers of $\tilde{V}$. Since the off-diagonal elements $(i \neq j)$ of the exchange integrals $V_{i j, j i}$ are small for localized basis functions, the main effect of the second term in Eq. (44) is to cancel the self-interaction in the first term.

It is not straightforward to anticipate the quality of a many-body calculation based on the effective interaction (43) as compared to the full interaction (3). Clearly, if we include all Feynman diagrams in $\Sigma$, we obtain the exact result when the full interaction (3) is used, while the use of the effective interaction (43) would yield an approximate result. The quality of this approximate result would then depend on the basis set, becoming better the more localized the basis functions and equal to the exact result in the limit of completely localized delta functions where only the direct Coulomb integrals $V_{i j, i j}$ will be non-zero. 
However, when only a subset of all diagrams are included in $\Sigma$ the situation is different: In the $G W$ approximation for instance, only one diagram per order (in $\hat{V}$ ) is included, and thus cancellation of self-interaction does not occur when the full interaction is used. On the other hand the effective interaction (44) is self-interaction free (in the orbital basis) by construction. The situation can be understood by considering the lowest order case. There are only two first order diagrams - the Hartree and exchange diagrams - and each cancel the self-interaction in the other. More generally, the presence of self-interaction in an incomplete perturbation expansion can be seen as a violation of identities of the form $\left\langle\cdot\left|c_{k \sigma^{\prime}}^{\dagger} \cdots c_{i \sigma} c_{i \sigma} \cdots c_{j \sigma^{\prime \prime}}\right| \cdot\right\rangle=0$, when not all Wick contractions are evaluated. Such expectation values will correctly vanish when the effective interaction is used because the prefactor of the $c_{i \sigma} c_{i \sigma}$ operator, $\tilde{V}_{i \sigma, i \sigma}$, is zero. The effect of self-interaction errors in (non-self consistent) $G W$ calculations was recently studied for a hydrogen atom (Nelson et al. 2007).

For a quantitative estimate of the quality of $G W$ calculations based on $\hat{V}_{\text {eff }}$ we refer to Thygesen and Rubio 2008.

\subsection{Non-equilibrium $G W$ self-energy}

It is useful to split the full many-body self-energy into its Hartree and exchange-correlation parts

$$
\Sigma\left(\tau, \tau^{\prime}\right)=\Sigma_{h}\left(\tau, \tau^{\prime}\right)+\Sigma_{x c}\left(\tau, \tau^{\prime}\right) .
$$

The Hartree term is local in time and can be written $\Sigma_{h}\left(\tau, \tau^{\prime}\right)=\Sigma_{h}(\tau) \delta_{\mathcal{C}}\left(\tau, \tau^{\prime}\right)$ where $\delta_{\mathcal{C}}$ is a delta function on the Keldysh contour. The xc- self-energy is nonlocal in time and contains all the complicated many-body effects. In the $G W$ approximation the xc- self-energy is written as a product of the Green's function, $G$, and the screened interaction, $W$. Usually $W$ is calculated in the random-phase approximation (RPA), and it has been found that improving $W$ beyond RPA has little effect on the resulting $G W$ self-energy (Verdozzi et al. 1995).

With the effective interaction (43) the screened interaction, $W$, and the polarization function, $P$, are reduced from four- to two-index functions. For notational simplicity we absorb the spin index into the orbital index, i.e. $(i \sigma) \rightarrow i$ (but we do not neglect it). The expression for the contour-ordered 


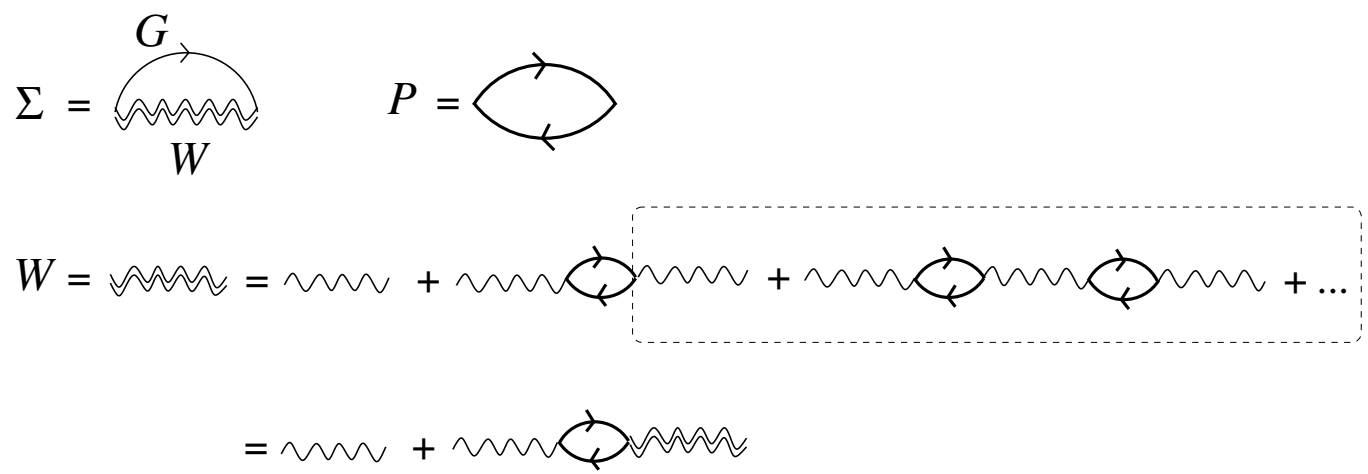

Figure 3: Feynman diagrams for the $G W$ self-energy in terms of the Green's function $(G)$, the screened interaction $(W)$, and the polarization bubble $(P)$. All quantities are functions of two Keldysh times, and two basis function indices. Integration/summation over internal times/indices is implied.

$G W$ self-energy in terms of contour-ordered quantities then read

$$
\begin{aligned}
\Sigma_{G W, i j}\left(\tau, \tau^{\prime}\right) & =i G_{i j}\left(\tau, \tau^{\prime+}\right) W_{i j}\left(\tau, \tau^{\prime}\right) \\
W_{i j}\left(\tau, \tau^{\prime}\right) & =\tilde{V}_{i j} \delta_{\mathcal{C}}\left(\tau, \tau^{\prime}\right)+\sum_{k l} \int_{\mathcal{C}} \mathrm{d} \tau_{1} \tilde{V}_{i k} P_{k l}\left(\tau, \tau_{1}\right) W_{l j}\left(\tau_{1}, \tau^{\prime}\right) \\
P_{i j}\left(\tau, \tau^{\prime}\right) & =-i G_{i j}\left(\tau, \tau^{\prime}\right) G_{j i}\left(\tau^{\prime}, \tau\right) .
\end{aligned}
$$

It is important to notice that in contrast to the conventional real-space representation of the $G W$ self-energy, the spin-dependence cannot be neglected when the effective interaction is used. The reason for this is that $\tilde{V}$ is spin-dependent and consequently the spin off-diagonal elements of $W$ will influence the spin-diagonal elements of $G, \Sigma$, and $P$. A diagrammatic representation of the $G W$ approximation is shown in Fig. 4.

As they stand, equations (46)-(48) involve quantities of the whole system (leads and central region). However, since $\tilde{V}_{i j}$ is non-zero only when $i, j \in$ $C$, it follows from Eq. (47), that $W$ and hence $\Sigma$ also have this structure. Consequently, the subscript $C$ can be directly attached to each quantity in Eqs. (46)-(48), however, for the sake of generality and notational simplicity we shall not do so at this point. It is, however, important to realize that the GF appearing in the $G W$ equations includes the self-energy due to the leads.

Using the Langreth conversion rules the retarded and lesser $G W$ selfenergies become (on the time axis),

$$
\begin{aligned}
& \Sigma_{G W, i j}^{r}(t)=i G_{i j}^{r}(t) W_{i j}^{>}(t)+i G_{i j}^{<}(t) W_{i j}^{r}(t) \\
& \Sigma_{G W, i j}^{</>}(t)=i G_{i j}^{</>}(t) W_{i j}^{</>}(t),
\end{aligned}
$$


where we have used the variable $t$ instead of the time difference $t^{\prime}-t$. For the screened interaction we obtain (in frequency space),

$$
\begin{aligned}
W^{r}(\omega) & =\tilde{V}\left[I-P^{r}(\omega) \tilde{V}\right]^{-1} \\
W^{</>}(\omega) & =W^{r}(\omega) P^{</>}(\omega) W^{a}(\omega) .
\end{aligned}
$$

where all quantities are matrices in the indices $i, \sigma$ and matrix multiplication is implied. Notice that the spin off-diagonal part of $\tilde{V}$ will affect the spindiagonal part of $W^{r}$ through the matrix inversion.

Finally, the real-time components of the irreducible polarization become

$$
\begin{aligned}
P_{i j}^{r}(t) & =-i G_{i j}^{r}(t) G_{j i}^{<}(-t)-i G_{i j}^{<}(t) G_{j i}^{a}(-t) \\
P_{i j}^{</>}(t) & =-i G_{i j}^{</>}(t) G_{j i}^{>/<}(-t) .
\end{aligned}
$$

From their definitions it is clear that both the polarization and the screened interaction obey the relations $P_{i j}^{a}(\omega)=P_{j i}^{r}(-\omega)$ and $W_{i j}^{a}(\omega)=W_{j i}^{r}(-\omega)$, while for the self-energy and GFs we have $\Sigma_{G W}^{a}(\omega)=\Sigma_{G W}^{r}(\omega)^{\dagger}$ and $G^{a}(\omega)=$ $G^{r}(\omega)^{\dagger}$. In addition all quantities fullfill the general identity $X^{>}-X^{<}=$ $X^{r}-X^{a}$. We mention that the nonequilibrium $G W$ approximation has previously been used to study band-gap renormalization in excited GaAs (Spataru et al. 2004).

In deriving Eqs. $(51,52)$ we have made use of the conversion rules $\delta_{\mathcal{C}}^{</>}\left(t, t^{\prime}\right)=$ 0 and $\delta_{\mathcal{C}}^{r / a}\left(t, t^{\prime}\right)=\delta\left(t-t^{\prime}\right)$. With these definitions the applicability of the Langreth rules can be extended to functions containing delta functions on the contour. Notice, however, that with these definitions relation (25) does not hold for the delta function. The reason why the delta function requires a separate treatment is that the standard Langreth rules are derived under the assumption that all functions on the contour are well behaved, e.g. not containing delta functions.

We stress that no spin symmetry has been assumed in the above $G W$ equations. Indeed by reintroducing the spin index, i.e. $i \rightarrow(i \sigma)$ and $j \rightarrow$ $\left(j \sigma^{\prime}\right)$, it is clear that spin-polarized calculations can be performed by treating $G_{\uparrow \uparrow}$ and $G_{\downarrow \downarrow}$ independently.

Within the $G W$ approximation the full interaction self-energy is given by

$$
\Sigma\left(\tau, \tau^{\prime}\right)=\Sigma_{h}\left(\tau, \tau^{\prime}\right)+\Sigma_{G W}\left(\tau, \tau^{\prime}\right)
$$

where the $G W$ self-energy can be further split into an static exchange and a dynamic correlation part,

$$
\Sigma_{G W}\left(\tau, \tau^{\prime}\right)=\Sigma_{x} \delta_{\mathcal{C}}\left(\tau, \tau^{\prime}\right)+\Sigma_{\text {corr }}\left(\tau, \tau^{\prime}\right)
$$


Due to the static nature of $\Sigma_{h}$ and $\Sigma_{x}$ we have

$$
\Sigma_{h}^{</>}=\Sigma_{x}^{</>}=0 .
$$

The retarded components of the Hartree and exchange self-energies become constant in frequency space, and we have (note that for $\Sigma_{h}$ and $\Sigma_{x}$ we do not use the effective interaction (43))

$$
\begin{aligned}
& \Sigma_{h, i j}^{r}=-i \sum_{k l} G_{k l}^{<}(t=0) V_{i k, j l} \\
& \Sigma_{x, i j}^{r}=i \sum_{k l} G_{k l}^{<}(t=0) V_{i k, l j} .
\end{aligned}
$$

Due to (57), it is clear that Eq. (50) yields the lesser/greater components of $\Sigma_{\text {corr }}$. Since $\Sigma_{\text {corr }}\left(\tau, \tau^{\prime}\right)$ does not contain delta functions its retarded component can be obtained from the relation,

$$
\Sigma_{\text {corr }}^{r}(t)=\theta(-t)\left[\Sigma_{G W}^{>}(t)-\Sigma_{G W}^{<}(t)\right] .
$$

The separate calculation of $\Sigma_{x}^{r}$ and $\Sigma_{\text {corr }}^{r}$ from Eqs. (59),(60) as opposed to calculating their sum directly from Eq. (49), has two advantages: (i) It allows us to treat $\Sigma_{x}$, which is the dominant contribution to $\Sigma_{G W}$, at a higher level of accuracy than $\Sigma_{\text {corr }}$. (ii) We avoid numerical operations involving $G^{r}$ and $W^{r}$ in the time domain. For more detailed discussions of these points see appendices A and E of Thygesen and Rubio 2008.

\subsection{Non-equilibrium second Born approximation}

When screening and/or strong correlation effects are less important, as e.g. in the case of small, isolated molecules, the higher-order terms of the $G W$ approximation are small and it is more important to include all second order diagrams (Stan et al. 2006). The full second order approximation, often referred to as the second Born approximation (2B), is shown diagrammatically in Fig. 4. As we will use the $2 \mathrm{~B}$ for comparison with the $G W$ results we state the relevant expressions here for completeness.

On the contour the $2 \mathrm{~B}$ self-energy reads (with the effective interaction $(43))$

$$
\begin{aligned}
\Sigma_{2 B, i j}\left(\tau, \tau^{\prime}\right) & =\sum_{k l} G_{i j}\left(\tau, \tau^{\prime}\right) G_{k l}\left(\tau, \tau^{\prime}\right) G_{l k}\left(\tau^{\prime}, \tau\right) \tilde{V}_{i k} \tilde{V}_{j l} \\
& -\sum_{k l} G_{i k}\left(\tau, \tau^{\prime}\right) G_{k l}\left(\tau^{\prime}, \tau\right) G_{l j}\left(\tau, \tau^{\prime}\right) \tilde{V}_{i l} \tilde{V}_{j k}
\end{aligned}
$$


Notice that the first term in $\Sigma_{2 B}$ is simply the second order term of the $G W$ self-energy. From Eq. (61) it is easy to obtain the lesser/greater self-energies,

$$
\begin{aligned}
\Sigma_{2 B, i j}^{</>}(t) & =\sum_{k l} G_{i j}^{</>}(t) G_{k l}^{</>}(t) G_{l k}^{>/<}(-t) \tilde{V}_{i k} \tilde{V}_{j l} \\
& -\sum_{k l} G_{i k}^{</>}(t) G_{k l}^{>/<}(-t) G_{l j}^{</>}(t) \tilde{V}_{i l} \tilde{V}_{j k}
\end{aligned}
$$

where $t$ has been used instead of the time difference $t-t^{\prime}$. Since these second order contributions do not contain delta functions of the time variable, we can obtain the retarded self-energy directly from the Kramers-Kronig relation

$$
\Sigma_{2 B}^{r}(t)=\theta(-t)\left[\Sigma_{2 B}^{>}(t)-\Sigma_{2 B}^{<}(t)\right] .
$$

\section{CURRENT}

As mentioned earlier, the current flowing between the electrodes can be calculated from the Green's function of the central region. After a short introduction to the concept of conserving approximations in section 4.1, we present the relevant formulas for evaluating the current under non-equilibrium conditions. In section 4.3 we then derive a condition on the interaction self-energy which ensures charge conservation in the sense that the current is the same at the left and right interface between the central region and the leads. Finally, in section 4.4 we show that this condition is always fulfilled for the so-called conserving, or $\Phi$-derivable, self-energies.

\subsection{Conserving approximations}

Once a self-energy has been obtained the GF follows from the Dyson and Keldysh equations (32),(34). Any single-particle observable, such as the current or the density, can then be calculated from the GF. An important question is then whether the calculated quantities obey the fundamental conservation laws like charge- and energy conservation. In the context of modeling electron transport, the condition for charge conservation as expressed by the continuity equation

$$
\frac{\mathrm{d}}{\mathrm{d} t} n(\mathbf{r}, t)=-\nabla \cdot \mathbf{j}(\mathbf{r}, t)
$$

is obviously of particular interest.

Baym and Kadanoff (Baym and Kadanoff 1961, Baym 1962) showed that there exists a deep connection between the self-energy and the validity of the conservation laws. Precisely, any self-energy which can be written as a 
functional derivative, $\Sigma[G]=\delta \Phi[G] / \delta G$, where $\Phi[G]$ belongs to a certain class of functionals of $G$, produces a GF which automatically fulfills the basic conservation laws. A self-energy which can be obtained in this way is called $\Phi$-derivable. Since a $\Phi$-derivable self-energy depends on $G$, the Dyson equation must be solved self-consistently.

The exact $\Phi[G]$ can be obtained by summing over all skeleton diagrams constructed using the full $G$ as propagator. By a skeleton diagram we mean a closed diagram, i.e. no external vertices, containing no self-energy insertions. Practical approximations are then obtained by including only a subset of skeleton diagrams. Two examples of such approximations are provided by the $G W$ and second Born $\Phi$-functionals and associated self-energies which are illustrated in Fig. 4. Another example is provided by the Hartree-Fock approximation. Solving the Dyson equation self-consistently with one of these self-energies thus defines a conserving approximation in the sense of Baym.

The validity of the conservation laws for $\Phi$-derivable self-energies follows from the invariance of $\Phi$ under certain transformations of the Green's function. For example it follows from the closed diagramatic structure of $\Phi$ that the transformation

$$
G\left(\mathbf{r} \tau, \mathbf{r}^{\prime} \tau^{\prime}\right) \rightarrow e^{i \Lambda(\mathbf{r} \tau)} G\left(\mathbf{r} \tau, \mathbf{r}^{\prime} \tau^{\prime}\right) e^{-i \Lambda\left(\mathbf{r}^{\prime} \tau^{\prime}\right)},
$$

where $\Lambda$ is any scalar function, leaves $\Phi[G]$ unchanged. Using the compact notation $\left(\mathbf{r}_{1}, \tau_{1}\right)=1$, the change in $\Phi$ when the GF is changed by $\delta G$ can be written as $\delta \Phi=\int \mathrm{d} 1 \mathrm{~d} 2 \Sigma(1,2) \delta G\left(2,1^{+}\right)=0$, where we have used that $\Sigma=\delta \Phi[G] / \delta G$. To first order in $\Lambda$ we then have

$$
\begin{aligned}
\delta \Phi & =i \int \mathrm{d} 1 \mathrm{~d} 2 \Sigma(1,2)[\Lambda(2)-\Lambda(1)] G\left(2,1^{+}\right) \\
& =i \int \mathrm{d} 1 \mathrm{~d} 2\left[\Sigma(1,2) G\left(2,1^{+}\right)-G\left(1,2^{+}\right) \Sigma(2,1)\right] \Lambda(1) .
\end{aligned}
$$

Since this hold for all $\Lambda$ (by a scaling argument) we conclude that

$$
\int \mathrm{d} 2\left[\Sigma(1,2) G\left(2,1^{+}\right)-G\left(1,2^{+}\right) \Sigma(2,1)\right]=0 .
$$

This condition ensures the validity of the continuity equation (on the contour) at any point in space. In the following sections we derive and discuss this result in the framework of the transport model introduced in Section 2.

\subsection{Current formula}

When the coupling between the leads and the central region is established, a current will start to flow. The particle current from lead $\alpha$ into the central 


$$
\begin{aligned}
& \Phi_{\mathrm{GW}}=-\frac{1}{2} \Leftrightarrow-\frac{1}{4} \theta-\frac{1}{6} \Delta+\ldots \\
& \Sigma_{\mathrm{GW}}=\Delta+\Delta+\Delta+\ldots \\
& \Phi_{2 B}=\frac{1}{4}
\end{aligned}
$$

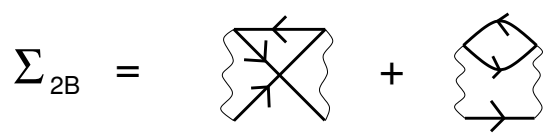

Figure 4: The $G W$ and second Born self-energies, $\Sigma_{G W}$ and $\Sigma_{2 B}$, can be obtained as functional derivatives of their respective $\Phi$-functionals, $\Phi_{G W}[G]$ and $\Phi_{2 B}[G]$. Straight lines represent the full Green's function, $G$, i.e. the Green's function in the presence of coupling to the leads and interactions. Wiggly lines represent the interactions. Reprinted with permission from Thygesen and Rubio, Phys. Rev. B 77, 115333 (2008). Copyright 2008 by the American Physical Society.

region is given by the time derivative of the number operator of lead $\alpha$ (Meir and Wingreen 1992),

$$
\begin{aligned}
I_{\alpha}(t) & =-i\left\langle\left[\hat{H}, \hat{N}_{\alpha}\right](t)\right\rangle \\
& =i \sum_{\substack{i \in \alpha \\
n \in C}} G_{n i}^{<}(t, t) h_{i n}-h_{n i} G_{i n}^{<}(t, t) .
\end{aligned}
$$

A simple diagrammatic argument shows that $(i \in \alpha, n \in C)$

$$
\begin{aligned}
G_{n i}\left(\tau, \tau^{\prime}\right) & =\sum_{\substack{j \in \mathcal{C} \\
m \in C}} \int_{\mathcal{C}} \mathrm{d} \tau_{1} G_{n m}\left(\tau, \tau_{1}\right) h_{m j} g_{0, j i}\left(\tau_{1}, \tau^{\prime}\right) \\
G_{i n}\left(\tau, \tau^{\prime}\right) & =\sum_{\substack{j \in \alpha \\
m \in C}} \int_{\mathcal{C}} \mathrm{d} \tau_{1} g_{0, i j}\left(\tau, \tau_{1}\right) h_{j m} G_{m n}\left(\tau_{1}, \tau^{\prime}\right) .
\end{aligned}
$$


Using Eq (20) we notice that Eq. (66) can be written as $i \operatorname{Tr}\left[A^{<}(t, t)\right]$ when $A$ is defined as in Eq. (92) with $B=G_{C}$ and $C=\Sigma_{\alpha}$. From the general result (93) it then follows that

$$
I_{\alpha}=\int \frac{\mathrm{d} \omega}{2 \pi} \operatorname{Tr}\left[\Sigma_{\alpha}^{<}(\omega) G_{C}^{>}(\omega)-\Sigma_{\alpha}^{>}(\omega) G_{C}^{<}(\omega)\right]
$$

where matrix multiplication is implied. By writing $I=\left(I_{L}-I_{R}\right) / 2$ we obtain an expression which is symmetric in the $L, R$ indices,

$$
I=\frac{i}{4 \pi} \int \operatorname{Tr}\left[\left(\Gamma_{L}-\Gamma_{R}\right) G_{C}^{<}+\left(f_{L} \Gamma_{L}-f_{R} \Gamma_{R}\right)\left(G_{C}^{r}-G_{C}^{a}\right)\right] \mathrm{d} \omega
$$

where we have suppressed the $\omega$ dependence and introduced the coupling strength of lead $\alpha, \Gamma_{\alpha}=i\left[\Sigma_{\alpha}^{r}-\Sigma_{\alpha}^{a}\right]$. We notice that when interactions are present, the integrals in Eqs. (68) and (67) will have contributions outside the bias window, $\mu_{L}<\omega<\mu_{R}$, because the conduction electrons can gain or lose energy by interacting with other electrons in the central region.

\subsection{Charge conservation}

Due to charge conservation in the steady state we expect that $I_{L}=-I_{R}=I$, i.e. the current flowing from the left lead to the molecule is the negative of the current flowing from the right lead to the molecule. Below we derive a condition for this specific form of particle conservation.

From Eq. (67) the difference between the currents at the left and right interface, $\Delta I=I_{L}+I_{R}$, is given by

$$
\Delta I=\int \frac{\mathrm{d} \omega}{2 \pi} \operatorname{Tr}\left[\left(\Sigma_{L}^{<}+\Sigma_{R}^{<}\right) G_{C}^{>}-\left(\Sigma_{L}^{>}+\Sigma_{R}^{>}\right) G_{C}^{<}\right]
$$

To obtain a condition for $\Delta I=0$ in terms of $\Sigma$ we start by proving the general identity

$$
\int \frac{\mathrm{d} \omega}{2 \pi} \operatorname{Tr}\left[\Sigma_{t o t}^{<}(\omega) G_{C}^{>}(\omega)-\Sigma_{t o t}^{>}(\omega) G_{C}^{<}(\omega)\right]=0 .
$$

To prove this, we insert $G^{</>}=G_{C}^{r} \Sigma_{\text {tot }}^{</>} G_{C}^{a}+\Delta^{</>}$(from Eq. (34)) in the left hand side of Eq. (70). This results in two terms involving $G^{r} \Sigma_{t o t}^{</>} G^{a}$ and two terms involving $\Delta^{</>}$. The first two terms contribute by

$$
\int \frac{\mathrm{d} \omega}{2 \pi} \operatorname{Tr}\left[\Sigma_{t o t}^{<} G^{r} \Sigma_{t o t}^{>} G^{a}-\Sigma_{t o t}^{>} G^{r} \Sigma_{t o t}^{<} G^{a}\right] .
$$


Inserting $\Sigma_{\text {tot }}^{>}=\Sigma_{\text {tot }}^{<}+\left(G^{a}\right)^{-1}-\left(G^{r}\right)^{-1}$ (we use that $\left(G^{a}\right)^{-1}-\left(G^{r}\right)^{-1}=$ $\left.\Sigma_{\text {tot }}^{r}-\Sigma_{\text {tot }}^{a}=\Sigma_{\text {tot }}^{>}-\Sigma_{\text {tot }}^{<}\right)$in this expression and using the cyclic invariance of the trace, it is straightforward to show that Eq. (71) vanishes. The two terms involving $\Delta^{</>}$contribute to the left hand side of Eq. (70) by

$$
\int \frac{\mathrm{d} \omega}{2 \pi} \operatorname{Tr}\left[\Sigma_{t o t}^{<}(\omega) \Delta^{>}(\omega)-\Sigma_{t o t}^{>}(\omega) \Delta^{<}(\omega)\right]
$$

As mentioned in section 2.3.2, $\Delta^{<}$and $\Delta^{>}$are always zero when interactions are present. In the case of non-interacting electrons we have $\Sigma_{\text {tot }}^{</>}=\Sigma_{L}^{</>}+$ $\Sigma_{R}^{</>}$, which vanish outside the band width the leads. On the other hand $\Delta^{</>}$is only non-zero at energies corresponding to bound states, i.e. states lying outside the bands, and thus we conclude that the term (72) is always zero.

From Eqs. (69) and (70) it then follows that

$$
\Delta I=\int \frac{\mathrm{d} \omega}{2 \pi} \operatorname{Tr}\left[\Sigma^{<}(\omega) G_{C}^{>}(\omega)-\Sigma^{>}(\omega) G_{C}^{<}(\omega)\right] .
$$

We notice that without any interactions particle conservation in the sense $\Delta I=0$ is trivially fulfilled since $\Sigma=0$. When interactions are present, particle conservation depends on the specific approximation used for the interaction self-energy, $\Sigma$.

\subsection{Charge conservation from $\Phi$-derivable self-energies}

One expects that there should be a connection between the condition for particle conservation as expressed by $\Delta I=0$ in Eq. (70), and the concept of a conserving approximation in the $\Phi$-derivable sense. Below we show that $\Delta I=0$ is always obeyed when the self-energy is $\Phi$-derivable.

We start by noting that Eq. (65) holds for any pair $G(1,2), \Sigma[G(1,2)]$ provided $\Sigma$ is of the $\Phi$-derivable form. In particular Eq. (65) does not assume that $G, \Sigma[G]$ fulfill a Dyson equation. Therefore, given any orthonormal, but not necessarily complete basis, $\left\{\phi_{i}\right\}$, and writing $G(1,2)=$ $\sum_{i j} \phi_{i}\left(\mathbf{r}_{1}\right) G_{i j}\left(\tau_{1}, \tau_{2}\right) \phi^{*}\left(\mathbf{r}_{2}\right)$ we get from Eq. (65) after integrating over $\mathbf{r}_{1}$,

$$
\sum_{j} \int_{\mathcal{C}} \mathrm{d} \tau^{\prime}\left[\Sigma_{i j}\left(\tau, \tau^{\prime}\right) G_{j i}\left(\tau^{\prime}, \tau^{+}\right)-G_{i j}\left(\tau^{-}, \tau^{\prime}\right) \Sigma_{j i}\left(\tau^{\prime}, \tau\right)\right]=0
$$

which in matrix notation takes the form

$$
\int_{\mathcal{C}} \mathrm{d} \tau^{\prime} \operatorname{Tr}\left[\Sigma\left(\tau, \tau^{\prime}\right) G\left(\tau^{\prime}, \tau^{+}\right)-G\left(\tau^{-}, \tau^{\prime}\right) \Sigma\left(\tau^{\prime}, \tau\right)\right]=0
$$


Here $\Sigma_{i j}$ is the self-energy matrix obtained when the diagrams are evaluated using $G_{i j}$ and the $V_{i j, k l}$ from Eq. (3). The left hand side of Eq. (75), which is always zero for a $\Phi$-derivable $\Sigma$, can be written as $\operatorname{Tr}\left[A^{<}(t, t)\right]$ when $A$ is given by Eq. (92) with $B=\Sigma$ and $C=G$. It then follows from the general result (93) and the condition (73) that current conservation in the sense $I_{L}=-I_{R}$ is always obeyed when $\Sigma$ is $\Phi$-derivable.

The above derivation of Eq. (75) relied on all the Coulomb matrix elements, $V_{i j, k l}$, being included in the evaluation of $\Sigma$. Thus the proof does not carry through if a general truncation scheme for the interaction matrix is used. However, in the special case of a truncated interaction of the form (43), i.e. when the interaction is a two-point function, Eq. (75) remains valid. To show this, it is more appropriate to work entirely in the matrix representation and thus define $\Phi\left[G_{i j}\left(\tau, \tau^{\prime}\right)\right]$ as the sum of a set of skeleton diagrams evaluated directly in terms $G_{i j}$ and $\tilde{V}_{i j}$. With the same argument as used in Eq. (64), it follows that $\Phi$ is invariant under the transformation

$$
G_{i j}\left(\tau, \tau^{\prime}\right) \rightarrow e^{i \Lambda_{i}(\tau)} G_{i j}\left(\tau, \tau^{\prime}\right) e^{-i \Lambda_{j}\left(\tau^{\prime}\right)},
$$

where $\Lambda$ is now a discrete vector. By adapting the arguments following Eq. (64) to the discrete case we arrive at Eq. (65) with the replacements $\mathbf{r}_{1} \rightarrow i$ and $\mathbf{r}_{2} \rightarrow j$ and with the integral replaced by a discrete sum over $j$. Summing also over $i$ leads directly to Eq. (75) which is the desired result.

To summarize, we have shown that particle conservation in the sense $I_{L}=-I_{R}$, is obeyed whenever a $\Phi$-derivable self-energy is used and either (i) all Coulomb matrix elements $V_{i j, k l}$ or (ii) the truncated two-point interaction of Eq. (43), are included in the calculation of $\Sigma$.

\subsubsection{Example: Transport through a single level}

As an illustrative example we consider transport through an Anderson impurity level connected to wideband leads, i.e. the retarded lead self-energies are taken to be frequency-independent equal to $i \Gamma$. The Hamiltonian of the central region reads

$$
\hat{H}_{C}=\varepsilon_{c} c^{\dagger} c+U n_{\uparrow} n_{\downarrow} .
$$

where $\varepsilon_{c}$ is the non-interacting energy and $U$ is the correlation energy.

In Figure 5 we show the current-voltage curve calculated from Eq. (68) using the selfconsistent HF, selfconsistent $G W$, and non-selfconsistent (NSC) $G W$ approximations. The parameters used are given in the figure caption. In the NSC calculation (referred to as $G_{0} W_{0}$ ) we use the self-consistent HartreeFock GF to evaluate the $G W$ self-energy. With this NSC self-energy we solve the Dyson's equation to obtain a NSC Green's function which is used to 


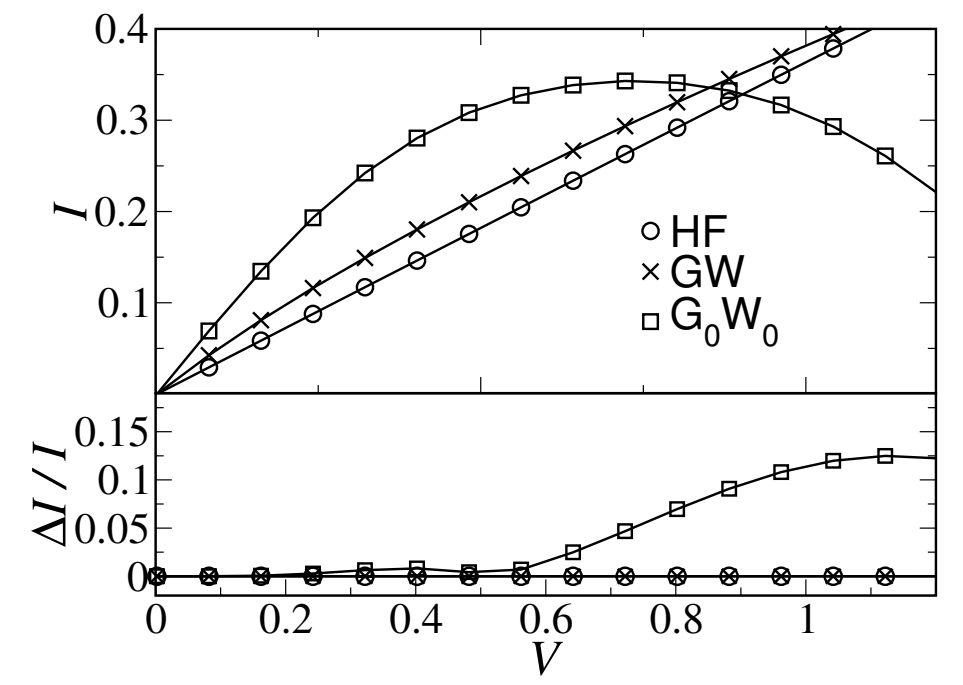

Figure 5: Current-voltage characteristic for an Anderson impurity level with $\Gamma=0.65, \varepsilon_{c}=-4, U=4$. At finite bias, the non-selfconsistent $G_{0} W_{0}\left[G_{\mathrm{HF}}\right]$ approximation yields different currents at the left and right interfaces $(\Delta I \neq 0)$ which means that charge conservation is violated. Moreover, $G_{0} W_{0}$ predicts significant negative differential conductance for $V>0.8$. In contrast, the $\Phi$-derivable $\mathrm{HF}$ and $G W$ self-energies both conserve charge.

calculate the current. This "one-shot" approach is not conserving, and as a result the currents calculated in the left and right leads from Eq. (67) are not guaranteed to coincide. From the lower panel of figure 5 it can be seen that the $G_{0} W_{0}$ self-energy does indeed violate charge conservation, and moreover leads to unphysical negative differential conductance for $V>0.8$. On the other hand the selfconsistent $\mathrm{HF}$ and $G W$ approximations both conserve charge.

At this point we mention that the role of self-consistency in $G W$ calculations has been much debated in the literature where it has been argued that $G_{0} W_{0}$, with $G_{0}$ being the DFT Green's function, produces better band structures and band gaps than self consistent $G W$. The present example clearly demonstrates that, regardless of the performance of $G W$ for band structure calculations, self-consistency is fundamental in nonequilibrium situations. 


\section{TWO-LEVEL MODEL}

In this section we apply the general formalism presented in the preceding sections to a generic two-level model of a molecular junction. In this model the molecule is represented by its highest occupied (HOMO) and lowest unoccupied (LUMO) orbitals and the leads are represented by one-dimensional tight-binding chains. With the aim of identifying universal trends we compare Hartree, Hartree-Fock, and $G W$ calculations for the spectrum and $I V$ characteristic. Not surprisingly the Hartree and HF results show large systematic differences due to the self-interaction errors in the Hartree potential. More interestingly, the dynamic correlations can have a large impact on both the spectrum and $I V$ leading to significant deviations between the $G W$ and HF results - in particular at finite bias.

In section 5.1 we introduce the two-level model. In section 5.2 we study how dynamic screening effects influence the equilibrium position of the HOMO and LUMO levels both in the case of weak and strong coupling between molecule and the leads. In section 5.3 we consider the nonequilibrium transport properties of the model and explain the features of the $I V$ curves in terms of the variation of the HOMO and LUMO positions as a function of the bias voltage.

\subsection{Hamiltonian}

The model consists of two electronic levels coupled to two semi-infinite 1D tight binding chains with nearest neighbor hopping, see Figure 6 . The levels represent the HOMO and LUMO states of a molecule and the TB chains represent metallic leads. Electron-electron interactions on the molecule, and between the molecule and the first site of the chains are included. The Hamiltonian of the two-level model reads

$$
\hat{H}=\hat{h}_{l}+\hat{h}_{r}+\hat{h}_{\text {mol }}+\hat{h}_{\text {coup }}+\hat{U}_{\text {mol }}+\hat{U}_{\text {ext }} .
$$

Notice that we use a notation different from the canonical $(L, C, R)$-notation introduced in section 2. This is because of the requirement that all interactions must be contained in the central region. Due to the interactions between the molecule and first sites of the leads, this implies that the central region should at least comprise the molecule and the first two sites of the leads. We enumerate the sites of the TB leads from $-\infty$ to -1 (left lead), and from 1 to $\infty$ (right lead). Thus $\hat{h}_{l}$ reads

$$
\hat{h}_{l}=\sum_{i=-\infty}^{-1} \sum_{\sigma=\uparrow, \downarrow} t\left(c_{i \sigma}^{\dagger} c_{i+1 \sigma}+c_{i+1 \sigma}^{\dagger} c_{i \sigma}\right)
$$



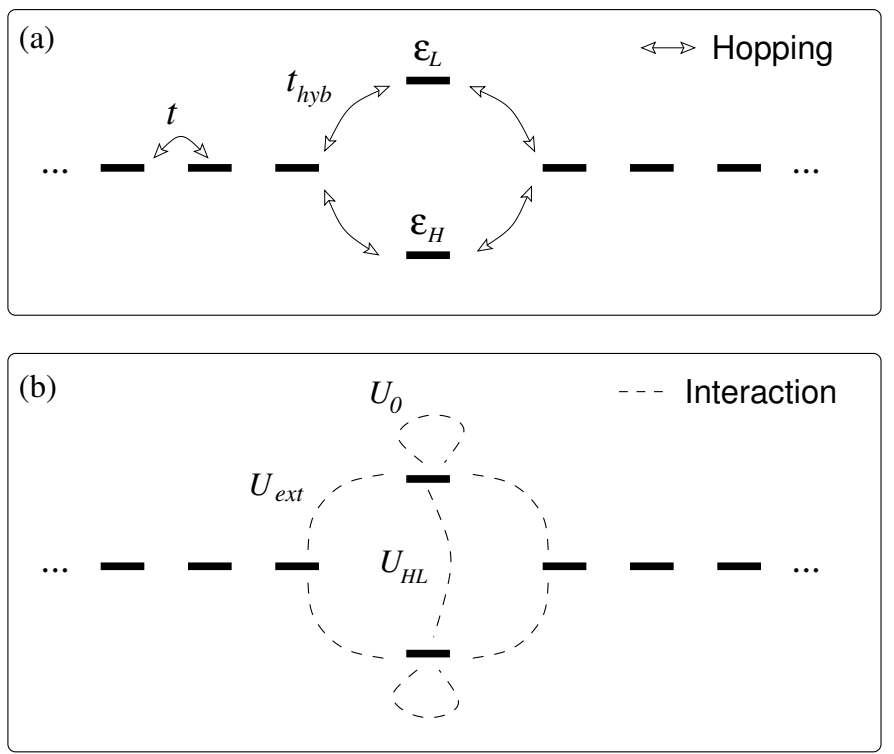

Figure 6: The two-level model used to describe the HOMO and LUMO levels of a molecule couped to metallic leads. (a) The one-particle hopping matrix elements. (b) The electron-electron interactions. The interactions can be divided into intra-molecule interactions $\left(U_{0}\right.$ and $\left.U_{H L}\right)$ and metal-molecule interactions $\left(U_{e x t}\right)$.

with a similar expression for $\hat{h}_{r}$. The non-interacting part of the molecule's Hamiltonian reads

$$
\hat{h}_{m o l}=\sum_{\alpha=H, L} \sum_{\sigma=\uparrow, \downarrow} \xi_{\alpha} d_{\alpha \sigma}^{\dagger} d_{\alpha \sigma}
$$

and the coupling is given by

$$
\hat{h}_{\text {coup }}=\sum_{\alpha=H, L} \sum_{\sigma=\uparrow, \downarrow} t_{h y b}\left(c_{1 \sigma}^{\dagger} d_{\alpha \sigma}+d_{\alpha \sigma}^{\dagger} c_{1 \sigma}+c_{-1 \sigma}^{\dagger} d_{\alpha \sigma}+d_{\alpha \sigma}^{\dagger} c_{-1 \sigma}\right)
$$

For clarity we use $c$-operators for the lead sites and $d$-operators for the HOMO/LUMO levels of the molecule.

The interactions are given by

$$
\begin{aligned}
\hat{U}_{\text {mol }} & =U_{0}\left(\hat{n}_{H \uparrow} \hat{n}_{H \downarrow}+\hat{n}_{L \uparrow} \hat{n}_{L \downarrow}\right)+U_{H L} \hat{n}_{H} \hat{n}_{L} \\
\hat{U}_{\text {ext }} & =U_{\text {ext }}\left(\hat{n}_{1} \hat{n}_{H}+\hat{n}_{1} \hat{n}_{L}+\hat{n}_{-1} \hat{n}_{H}+\hat{n}_{-1} \hat{n}_{L}\right)
\end{aligned}
$$

where e.g. $\hat{n}_{H}=d_{H \uparrow}^{\dagger} d_{H \uparrow}+d_{H \downarrow}^{\dagger} d_{H \downarrow}$ is the number operator of the HOMO level, and $\hat{n}_{1}$ is the number operator of the first site of the right lead. 
We set the Fermi level to zero corresponding to half-filled bands. In general, we write $\xi_{L}=\xi_{H}+\Delta_{0}$, i.e. we use the non-interacting energy gap as a free parameter. The occupation of the molecule can then be controlled by adjusting $\xi_{H}$.

\subsection{Renormalization of molecular levels by dynamic screening}

In the low-bias regime, the transport properties are to a large extent determined by the position of the HOMO and LUMO levels relative to the Fermi energy of the metal electrodes. For this reason we shall first consider how the HOMO and LUMO position are affected by the interactions in the zero bias limit. The material presented in this section is part of ongoing work which will be published elsewhere.

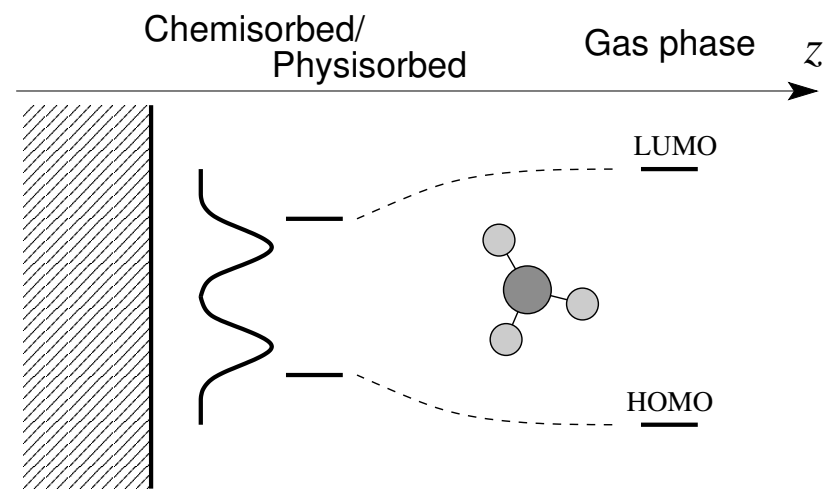

Figure 7: As a molecule approaches a metal surface its HOMO-LUMO gap is reduced by image-charge formation in the metal. If the molecule-metal bond is sufficiently strong (chemisorption) dynamic charge transfer between molecule and metal can give rise to additional reduction of the gap. These renormalization effects requires a dynamic, i.e. frequency dependent, selfenergy and thus cannot be described within the single-particle picture.

When a molecule is brought into contact with a metal surface a number of different mechanisms will affect the position of the molecule's energy levels. First, the levels are shifted by the electrostatic potential outside the surface. Second, hybridization effects shift and broaden the levels into resonances with finite life-times. For our model, the resonance width due to coupling to the leads is $\Gamma \approx\left|t_{\text {hyb }}\right|^{2} / t$.

Both the shift due to the surface potential and the hybridization are single-particle effects which can be described at a mean-field level such as 
Kohn-Sham (KS) or Hartree-Fock (HF) theory. On the other hand, correlation effects can renormalize the molecular spectrum in a way that cannot be described within a single-particle picture. Correlation effects are generally small for isolated molecules where HF usually yields good spectra, however, they can become significant when the molecule is in contact with a surface. An important example is the Kondo effect, where electronic interactions qualitatively changes the molecule's spectrum by introducing a narrow peak at the metal Fermi level (Goldhaber 1998 et al., Costi et al. 1994, Thygesen and Rubio et al. 2008). In weakly correlated systems such as molecules with a closed shell structure adsorbed at a surface, the effect of electronic interactions is expected to be less dramatic. However, as we shall see below, dynamic screening at the molecule-metal interface can introduce significant reductions of the HOMO-LUMO gap, which in turn will influence the transport through the molecule. Such screening effects can be observed in photoemission- and electron tunneling spectroscopy (Johnson and Hulbert 1987, Kubatkin et al. 2003, Repp et al. 2005). Recently, first-principles $G W$ calculations for benzene physisorbed on graphite showed a HOMO-LUMO gap reduction of more than $3 \mathrm{eV}$ due to substrate polarization (Neaton et al. 2006). More empirical treatments of polarization/screening effects using a scissors operator on the DFT spectrum have recently been applied to transport in molecules (Quek et. al 2007 and Mowbray et al. 2008) and scanning tunneling microscopy simulations (Dubois et al. 2007). So far the theoretical studies have focused on weakly coupled (physisorbed) molecules where the gap renormalization is induced by substrate polarization. This is the situation studied in section 5.2.2 below. In section 5.2 .3 we consider the to the case of strongly coupled (chemisorbed) molecules.

\subsubsection{The quasi-particle picture}

In interacting many-electron systems the concept of a single-particle eigenenergy becomes meaningless. However, for weakly correlated systems the concept can still be maintained due to the long life-time of certain states of the form $c_{m}^{\dagger}\left|\Psi_{0}\right\rangle$ (for $\phi_{m}$ unoccupied) or $c_{m}\left|\Psi_{0}\right\rangle$ (for $\phi_{m}$ occupied). These quasiparticle (QP) states describes the many-body $N$-particle groundstate with and added electron (hole). The energy of the QP states relative to the groundstate energy, is given by the spectral function, $A_{m}(\varepsilon)=-\operatorname{Im} G_{m m}^{r}(\varepsilon)$, where $G^{r}$ is the retarded Green's function. For weakly correlated systems $A_{m}(\varepsilon)$ will be peaked at the QP energy, $\varepsilon_{m}$, which is equivalent to saying that the QP has a long life-time. It is instructive to notice that the QP energies measures the cost of removing/adding an electron to the state $\left|\phi_{m}\right\rangle$ in the presence of interactions with the other electrons of the system. 
For non-interacting electrons the peaks in the spectral function coincide with the eigenvalues of the single-particle Hamiltonian. Meanfield theories like $\mathrm{KS}$ or $\mathrm{HF}$ includes interactions at a static level, i.e. the singleparticle eigenvalues correspond to the energy cost of adding/removing an electron when the state of all other electrons is kept fixed. This is the content of Koopman's theorem which states that (for $\phi_{m}$ occupied), $\varepsilon_{m}^{\mathrm{HF}}=$ $E\left[\Phi_{0}^{\mathrm{HF}}\right]-E\left[c_{m} \Phi_{0}^{\mathrm{HF}}\right]$, i.e. the $\mathrm{HF}$ eigenvalues correspond to unrelaxed removal/addition energies. In general, the other electrons will respond to the added electron/hole and this will shift, or renormalize, the HF energies. The size of this effect is expected to qualitatively follow the linear response function, $\chi$, which gives the change in the particle density when the system is subject to an external field,

$$
\delta n(\mathbf{r} ; \omega)=\int \mathrm{d} \mathbf{r} \chi\left(\mathbf{r}, \mathbf{r}^{\prime} ; \omega\right) v_{\text {ext }}\left(\mathbf{r}^{\prime} ; \omega\right) .
$$

This suggests a direct relation between the impact of dynamic relaxations, or screening, on the QP spectrum, and the static response function $\chi(\omega=0)$.

\subsubsection{Weak molecule-lead coupling}

We first consider the case of a weakly coupled, or physisorbed, molecule corresponding to small $t_{h y b}$. We use the following default parameter values: $t=10, U_{0}=4, U_{H L}=3, U_{\text {ext }}=2, t_{\text {hyb }}=0.3, \Delta_{0}=4$, which yield a resonance width of $\Gamma \approx 0.01$. In Fig. 8(left) we show the HOMO and LUMO positions as function of the interaction $U_{\text {ext }}$ as calculated using the $\mathrm{HF}$ and $G W$ approximations. As $U_{\text {ext }}$ is increased corresponding to the molecule approaching the surface, the $G W$ gap decreases while the HF gap remains unchanged. In the simplest picture the gap reduction is due to the interaction between the added/removed electron and its image charge in the metal. This effect is not present in the HF single-particle spectrum: According to Koopman's theorem the added/removed electron interacts with the HF groundstate of the neutral system which contains no image charge. For small $t_{h y b}$ where the molecule's levels are well defined in energy and localized it is possible to include the response of the metal to the added/removed electron by performing HF total energy calculation with constrained HOMO/LUMO occupation numbers. Denoting by $\Phi_{0}^{n}$ the minimizing Slater determinant with the constrain of $n$ excess electrons on the molecule, we can define constrained HF energy levels as the total energy difference

$$
\bar{\varepsilon}_{L / H}^{\mathrm{HF}}= \pm\left(E\left[\Phi_{0}^{ \pm 1}\right]-E\left[\Phi_{0}^{0}\right]\right) .
$$


From the very good agreement between $\bar{\varepsilon}_{n}^{\mathrm{HF}}$ and $\varepsilon_{n}^{G W}$ seen in Fig. $8 \mathrm{~b}$, we conclude that $G W$ includes the effect of relaxation or screening in the metal at the HF level. The situation is sketched in Fig. 10a.
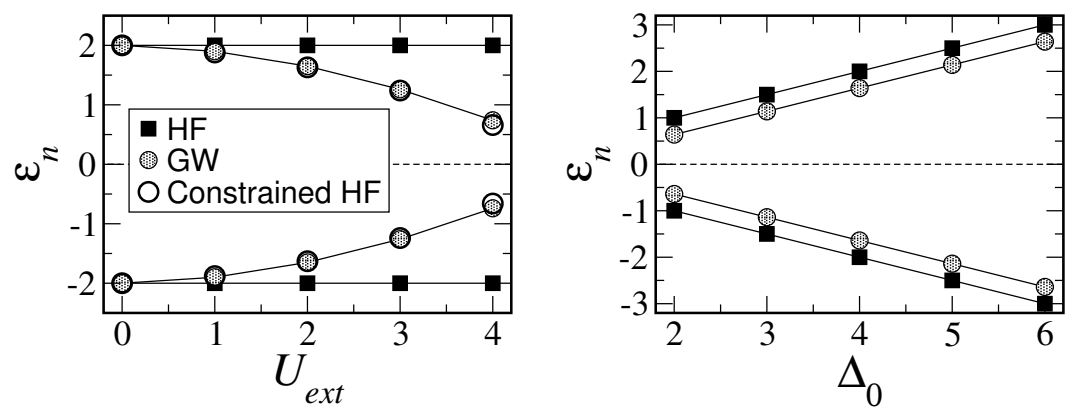

Figure 8: Position of the HOMO and LUMO levels as function of interaction strength $U_{\text {ext }}$ (left) and non-interacting gap $\Delta_{0}$ (right) for a weakly coupled molecule (small $\Gamma$ ). The $G W$ HOMO (LUMO) correspond to the HF energy cost of removing (adding) an electron to the molecule when the Slater determinant of the metal is allowed to relax. The gap reduction from (unrelaxed) HF to $G W$ is thus due to polarization of the metal. This gap reduction is independent of $\Delta_{0}$.

Koopman's theorem allow us to write the difference between the HF single-particle levels and the result of constrained total energies, as (in case of the LUMO) $\varepsilon_{L}^{\mathrm{HF}}-\bar{\varepsilon}_{L}^{\mathrm{HF}}=E\left[\Phi_{0}^{+1}\right]-E\left[d_{L}^{\dagger} \Phi_{0}^{0}\right]$. This energy difference has two contributions: A positive one from the interaction between the added electron and the induced density in the metal, and a negative one being the cost of forming the induced density. The classical image charge approximation in contrast assumes perfect screening in the metal and zero energy cost of polarizing the metal.

From the right panel of Fig. 8 it can be seen that the renormalization of the gap is independent on the intrinsic gap of the molecule. This is expected since the image-charge and its interaction with the added electron/hole is independent on the HOMO-LUMO positions.

According to the above, the size of the gap reduction for fixed $U_{\text {ext }}$ should depend on the polarizability of the metal. In Fig. 9(top) we show the dependence of the levels as function of $t$, i.e. the bandwidth of the metal. The effect is larger for small $t$ corresponding to a narrow band. This is easily undestood by noting that narrow bands have larger DOS at $\varepsilon_{F}$ which in turn implies a larger density response function. Indeed, the right panel of Fig. 9 

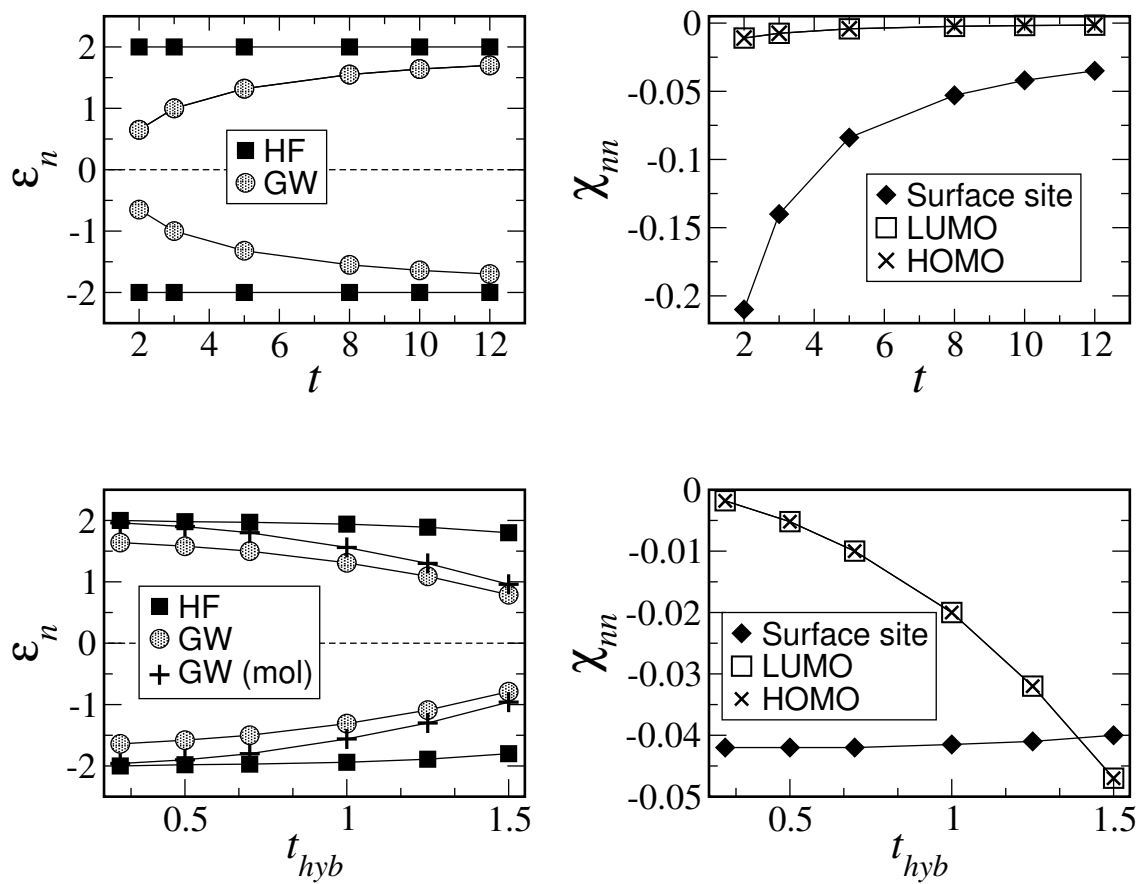

Figure 9: Left panels: Position of the molecule's HOMO and LUMO levels as a function of the metal bandwidth, $t$ and the hybridization strength, $t_{h y b}$, respectively. $G W(\mathrm{~mol})$ refers to a calculation where only the interactions internally on the molecule have been treated within $G W$. Right panels: Static linear response functions (RPA) $\left\langle\phi_{n}|\chi(\omega=0)| \phi_{n}\right\rangle$ for the HOMO, LUMO and terminal site of the TB chain. The reduction of the correlated gap relative to $\mathrm{HF}$ is due to polarization of the metal and, for large $t_{h y b}$, of the molecule itself. Default parameter values are the same as in Fig.8

shows the diagonal elements of the static (RPA) response function for the HOMO, LUMO and terminal site of the chain. The response function of the HOMO and LUMO is negligible for all values of $t$, while the response of the terminal site is significant and increases as $t$ is reduced.

\subsubsection{Strong molecule-lead coupling}

We now turn to the case of a strongly coupled, or chemisorbed, molecule corresponding to non-negligible $t_{h y b}$. In the bottom panel of Fig. 9 we show the center of the molecular resonances as a function of $t_{h y b}$. In addition to the $\mathrm{HF}$ and $G W$ values, we also show the result when only $\hat{U}_{\text {mol }}$ is treated at the $G W$ level while $\hat{U}_{\text {ext }}$ is treated within HF. This allows us to isolate the 
Physisorbed molecule
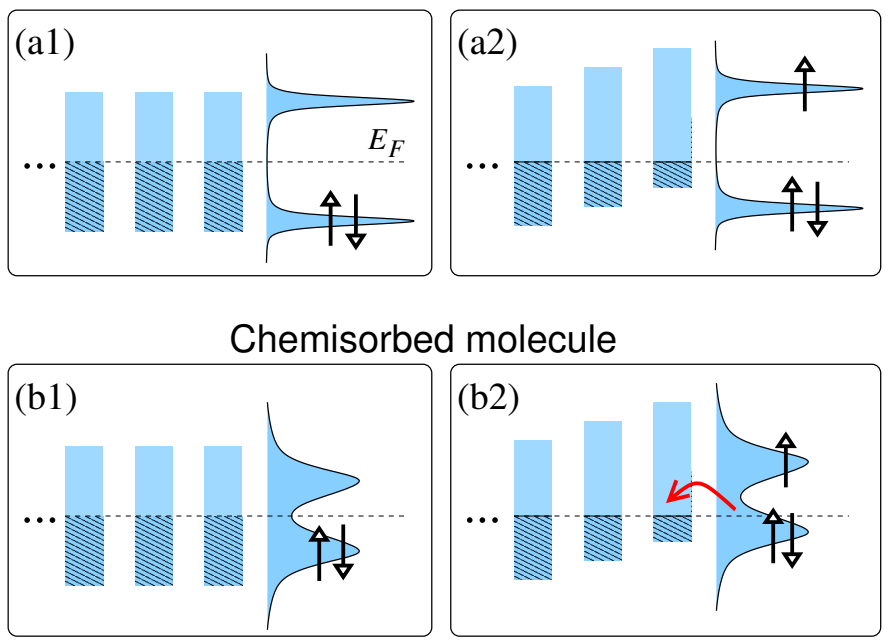

Figure 10: (a1) Groundstate of a physisorbed molecule at a metal surface. (a2) When an electron is added to the molecule, the metal is polarized. (b1) Groundstate of a chemisorbed molecule at a metal surface. (b2) When an electron is added to the LUMO the metal is polarized, and charge is transfered from the molecule to the metal. The screening effects stabilize the system with added/removed electron and this shift the occupied (unoccupied) quasi-particle levels up (down).

correlation effects induced by the intra-molecular interactions from those of the metal-molecule interactions.

Clearly, the correlated gap decrease relative to the HF gap as $t_{h y b}$ is increased. It is also clear that the coupling-dependent part of the gap reduction comes from the interactions internally on the molecule, while the reduction due to $\hat{V}_{\text {ext }}$ is largely independent of $t_{h y b}$. Since $\hat{V}_{\text {mol }}$ does not produce any renormalization of the levels of the free molecule (see the $t_{h y b} \rightarrow 0$ limit), the mechanism responsible for the gap reduction must involve the metal. From the lower right panel of Fig. 9, we see that the response functions of the HOMO and LUMO states increases with $t_{h y b}$ indicating the gap reduction due to $\hat{V}_{\text {mol }}$ is of a similar nature as the image charge effect, but with the molecule itself being polarized. The effect increases with $t_{h y b}$ because charge transfer between the molecule and the metal due to the external field from the added/removed electron, is larger when resonances are broad and have larger overlap with the metal Fermi level. The situation is sketched in Fig. 10b. 


\subsection{Nonequilibrium transport}

The analysis of the previous sections show that dynamic screening effects can have a large effect on the spectrum of the molecule in contact with leads. In this section we shall see that the application of a bias voltage leads to additional renormalization of the spectrum. For simplicity we limit the model to include intra-molecule interactions, i.e. we set $U_{\text {ext }}$ to zero. This means that reduction of the HOMO-LUMO gap due to image charge formation in the leads is not included. Whereas the presence of intra-molecule interactions did not have a large influence on the equilibrium positions of the HOMO and LUMO levels for small values of $\Gamma$ (see the $G W(\mathrm{~mol})$ result in Fig. 9), we will see that this is no longer true under finite bias conditions, where intramolecular screening is strongly enhanced and the life-times of the HOMO and LUMO levels can be significantly reduced due to QP scattering. Both effects lead to a reduction of the HOMO-LUMO gap as function of the bias voltage with a large impact in the calculated $I V$ curve.

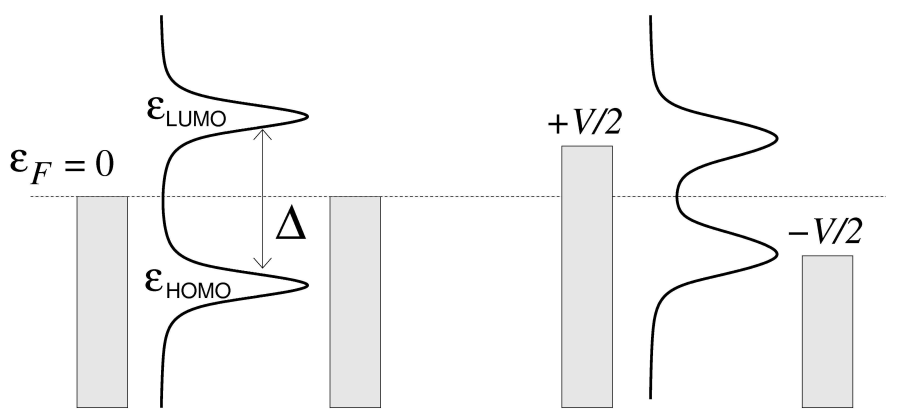

Figure 11: The HOMO and LUMO resonances of the two-level model under zero and non-zero bias voltage. As indicated the bias affects both the position and width of the resonances and this will in turn affect the $d I / d V$ curve. Reprinted with permission from Thygesen, Phys. Rev. Lett. 100, 166804 (2008). Copyright 2008 by the American Physical Society.

Throughout this section we use following parameters: $\Delta_{0}=2, U_{0}=2$, $U_{H L}=1.5, t=10$. By varying the one-particle energy $\xi_{0}$, we can control the equilibrium occupation of the molecule, $N_{\mathrm{el}}$. We consider the case of weak charge transfer to the molecule, i.e. $N_{\text {el }}$ ranges from 2.0 to 2.1, corresponding to $\varepsilon_{F}$ lying in the middle of the gap and slightly below the LUMO, respectively. The Fermi level is set to zero, and the bias is applied symmetrically, i.e. $\mu_{L}=V / 2$ and $\mu_{R}=-V / 2$. The situation is illustrated in figure 11 . 
In Fig. 12 we show the calculated $d I / d V$ curves (obtained by numerical differentiation of $I(V))$ for different values of $\Gamma$ and $N_{\mathrm{el}}$. We first notice that the $2 B$ and $G W$ approximations yield similar results in all the cases indicating that the higher order terms in the $G W$ self-energy are fairly small. For $\Gamma=1.0$, all methods yield qualitatively the same result. For even larger values of $\Gamma$ (not shown), and independently of $N_{\mathrm{el}}$, the results become even more similar. In this strong coupling limit, single-particle hybridization effects will dominate over the interactions and xc-effects are small.
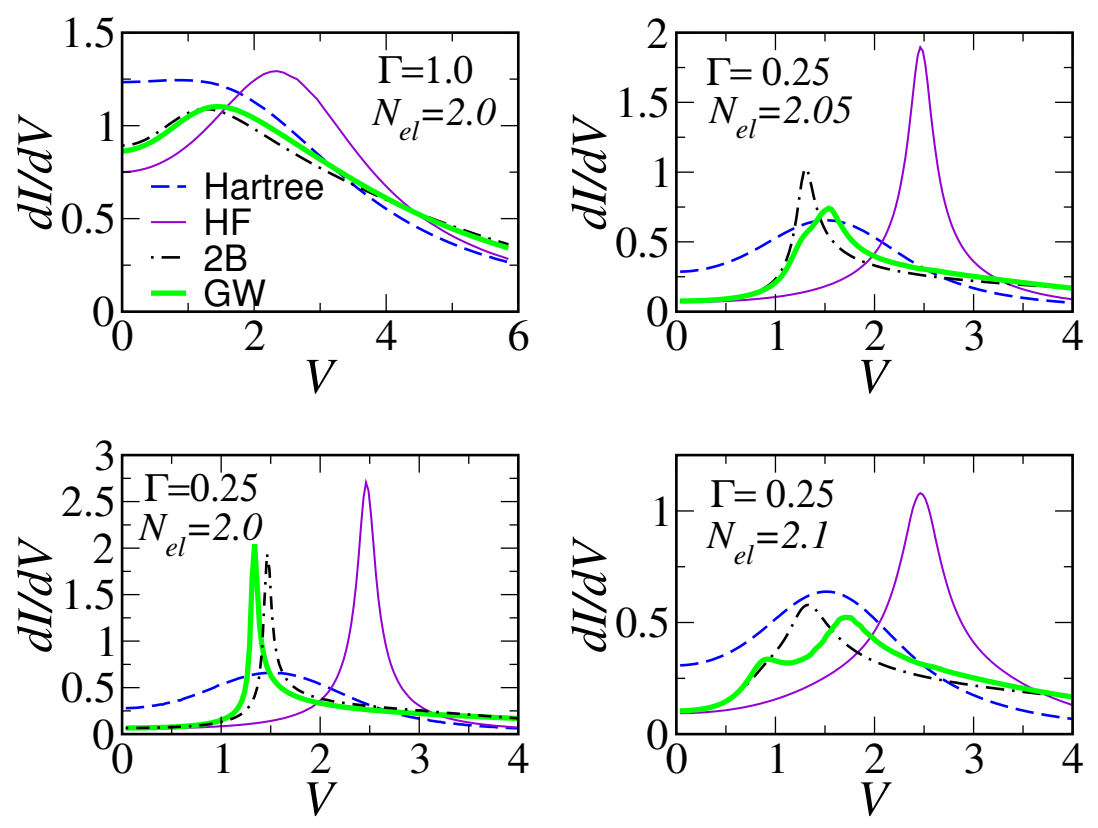

Figure 12: $d I / d V$ curves for different values of the tunneling strength $\Gamma$ and occupation of the molecule, $N_{\mathrm{el}}$. The curves are calculated using different approximations for the xc self-energy. Reprinted with permission from Thygesen, Phys. Rev. Lett. 100, 166804 (2008). Copyright 2008 by the American Physical Society.

Focusing on the $\Gamma=0.25$ case we see that the Hartree approximation severely overestimates the low-bias conductance. This is a consequence of the self-interactions (SI) contained in the Hartree potential which leads to an underestimation of the (equilibrium) HOMO-LUMO gap, see figure 13 for $V=0$. On the other hand the $\mathrm{HF}, 2 \mathrm{~B}$, and $G W$ methods lead to very similar conductances in the low-bias regime. This is consistent with the results of the previous section which showed that intra-molecular correlations do not 
renormalize the equilibrium $\mathrm{HF}$ gap much for small $\Gamma$. For $\Gamma=1.0$ the slightly larger conductance in $G W$ and $2 \mathrm{~B}$ is due to the slight reduction of the equilibrium gap.

We notice that the lower left graph $\left(\Gamma=0.25, N_{\mathrm{el}}=2.0\right)$ shows an interesting feature. Namely, the $\mathrm{HF}, 2 \mathrm{~B}$, and $G W$ curves all contain an anomalously strong conductance peak. Interestingly, the peak height is significantly larger than 1 which is the maximum conductance for a single level (the Anderson impurity model). Moreover, the full width at half maximum (FWHM) of the peak is only $\sigma_{\mathrm{HF}}=0.27$ and $\sigma_{2 \mathrm{~B} / G W}=0.12$, respectively, which is much smaller than the tunneling broadening of $2 \Gamma=0.5$. We note in passing that the peak looses intensity as $N_{\mathrm{el}}$ is increased, and that the Hartree approximation does not reproduce the narrow peak.

\subsubsection{Influence of bias on the HOMO and LUMO positions}

To understand the origin of the anomalous conductance peaks, we consider the evolution of the HOMO and LUMO positions as a function of the bias voltage, see Fig. 13 (the $2 \mathrm{~B}$ result is left out as it is similar to $G W$ ). Focusing on the upper panel of the figure (corresponding to $N_{\mathrm{el}}=2.0$ ), we notice a qualitative difference between the Hartree and the SI-free approximations: While the Hartree gap expands as the levels move into the bias window, the $\mathrm{HF}$ and $G W$ gaps shrink leading to a dramatic increase in current around $V=2.5$ and $V=1.3$, respectively. This is clearly the origin of the anomalous $d I / d V$ peaks. But why do the SI-free gaps shrink as the bias is raised?

Let us consider the change in the HOMO and LUMO positions when the bias $V$ is increased by $2 \delta V$. In general this change must be determined self-consistently, however, a "first iteration" estimate yields a change in the HOMO and LUMO occupations of $\delta n_{H} \approx-A_{H}(-V / 2) \delta V$ and $\delta n_{L} \approx$ $A_{L}(V / 2) \delta V$, respectively. Here $A_{H / L}$ is the spectral function, or equivalently the DOS, of the HOMO/LUMO levels. At the HF level this leads to

$$
\begin{aligned}
\delta \varepsilon_{H} & \approx\left[-U_{0} A(-V / 2)+2 U_{H L} A(V / 2)\right] \delta V \\
\delta \varepsilon_{L} & \approx\left[U_{0} A(V / 2)-2 U_{H L} A(-V / 2)\right] \delta V
\end{aligned}
$$

where $A=H_{H}+A_{L}$ is the total DOS on the molecule and we have used that $A_{H}(-V / 2) \approx A(-V / 2)$ and $A(V / 2) \approx A(V / 2)$. The factor 2 in front of $U_{H L}$ accounts for interactions with both spin channels. In the symmetric case $\left(N_{\mathrm{el}}=2.0\right)$ we have $A(-V / 2)=A(V / 2)$. Since $U_{0}<2 U_{H L}$ this means that $\delta \varepsilon_{H}>0$ and $\delta \varepsilon_{L}<0$, i.e. the gap is reduced as $V$ is raised. Moreover it follows that the gap reduction is largest when $A( \pm V / 2)$ is largest, that is, just when the levels cross the bias window. In the general case $\left(N_{\mathrm{el}} \neq 2.0\right)$ 

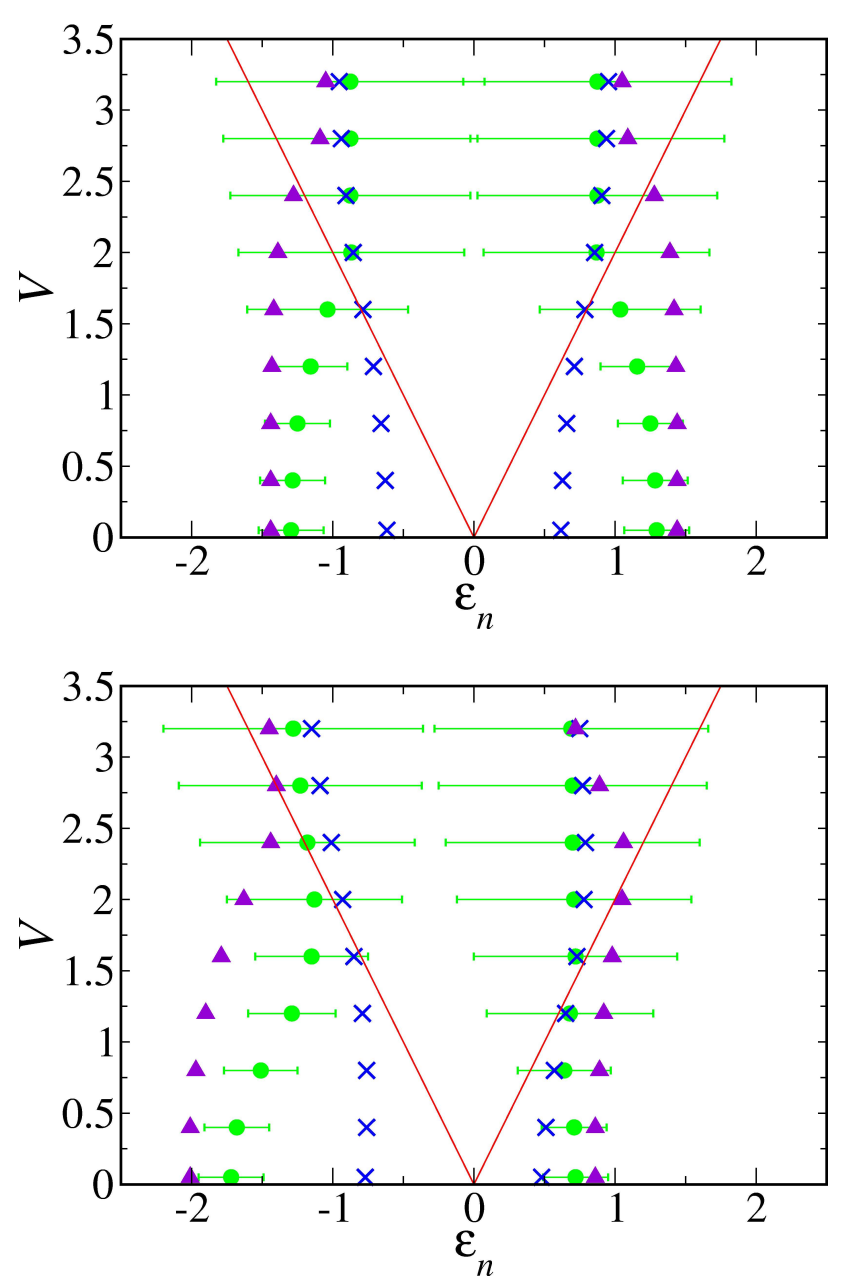

Figure 13: Position of the HOMO and LUMO levels as a function of the bias voltage for the Hartree (crosses), HF (triangles), and $G W$ (circles) approximations. The horizontal lines show the FWHM of the $G W$ resonances. The FWHM of the Hartree and HF resonances is $2 \Gamma$ independently of $V$. Notice the differences in the way the levels enter the bias window: The Hartree gap opens while the $\mathrm{HF}$ and $G W$ gaps close. In the upper graph $\Gamma=0.25, N_{\mathrm{el}}=2.0$ (symmetric case). In the lower graph $\Gamma=0.25, N_{\mathrm{el}}=2.1$. Reprinted with permission from Thygesen, Phys. Rev. Lett. 100, 166804 (2008). Copyright 2008 by the American Physical Society.

the direction of the shift depends on the relative magnitude of the DOS at the two bias window edges: a level will follow the edge of the bias window if the other level does not intersect the bias edge. It will move opposite to the 
bias, i.e. into the bias window, if the other level is close to the bias window edge. This is effect clearly seen in the lower graph of Fig. 13 (triangles). Thus the gap closing mechanism has the largest impact on the $d I / d V$ curve when the HOMO and LUMO levels hit the bias window simultaneously. Moreover, the effect is stronger the larger $U_{H L} / U_{0}$, and the smaller $\Gamma$ (the maximum in the DOS is $\sim 1 / \Gamma)$. At the Hartree level, Eqs. (86) and (87) are modified by replacing $U_{0}$ by $2 U_{0}$ due to self-interaction. This leads to an effective pinning of the levels to the bias window which tends to open the gap as $V$ is increased, see Fig. 13 (crosses).

The above analysis shows why the HF gap is reduced as the levels hit the bias window. Interestingly, the bias-driven gap reduction is even stronger in $G W$ and as a consequence the $G W$ conductance peak occurs at much lower bias $(V=1.5)$ than the HF peak $(V=2.5)$. Part of the downshift of the $G W$ conductance peak can be explained from the smaller $G W$ equilibrium gap. Indeed, for $V=0$ the HF gap is $\sim 0.3$ larger than the $G W$ gap. However, this effect alone cannot account for the large down-shift.

The reason for the bias induced reduction of the $G W$ gap is two-fold: First, intra-molecular screening effects are enhanced as the chemical potentials move closer to the molecular levels and increase the susceptibility of the levels. This is analogue to the (equilibrium) situation of increasing $\Gamma$ shown in lower panels of figure 9. The susceptibility of a molecular level is roughly given by the magnitude of the level's DOS at $E_{F}$ (or chemical potentials). In the latter case this is achieved by broadening the resonances; in the former case by bringing the chemical potential(s) closer to the levels. Secondly, the rate of QP scattering, i.e. the rate at which the initial state $c_{i}^{\dagger}\left|\Psi_{0}\right\rangle$ is destroyed due to electron-electron interactions, increases with the bias. This follows from Fermi's Golden rule by realizing that the number of available final states of the form $c_{f}^{\dagger}\left|\Psi_{0}\right\rangle$ having the same energy as the initial state, increases with bias. The enhanced QP scattering reduces the life-time of the HOMO and LUMO QPs, which is equivalent to a broadening of the molecular resonances.

The full width at half maximum (FWHM) of the $G W$ resonances is indicated by horizontal lines in Fig. 13. For low bias, the width of the $G W$ resonances is the same as the width of the Hartree and HF resonances. The latter is determined by the coupling to leads and equals $2 \Gamma=0.5$ independently of the bias. According to Fermi-liquid theory, QP scattering at the Fermi level is strongly suppressed in the ground state, i.e. $\operatorname{Im} \Sigma_{i i}\left(\varepsilon_{F}\right)=0$ for $V=0$ (recall that $\operatorname{Im} \Sigma$ is inversely proportinal to the life time). However, as the bias is raised the phase space available for QP scattering is enlarged and $\operatorname{Im} \Sigma$ increases accordingly. As a result of the additional level broadening, $A( \pm V / 2)$ increases more rapidly as a function of $V$. According to 
Eqs. $(86,87)$, this will accelerate the gap closing reduction already at the HF level. Finally, we notice that the long, flat tails seen in the $d I / d V$ of the $G W / 2 \mathrm{~B}$ calculations are also a result of the spectral broadening due to QP scattering.

\section{PROTOTYPE MOLECULAR JUNCTIONS: $\mathbf{C}_{6} \mathbf{H}_{6}$ and $\mathbf{H}_{2}$}

In this section we combine the $G W$ scheme with a Wannier function (WF) basis set to study electron transport through two prototypical junctions, namely a benzene molecule coupled to featureless leads and a hydrogen molecule between two semi-infinite Pt chains. In section 6.1 we briefly present the computational scheme. In the following two sections we analyze the energy spectrum and transport properties of the benzene junction. Finally in section 6.4 we present results for the $I V$ curve of the $\mathrm{Pt}_{-} \mathrm{H}_{2}-\mathrm{Pt}$ junction.

\subsection{Computational details}

Below we review our computational scheme for $G W$ transport calculations in a WF basis discussed in detail in Thygesen and Rubio 2008. In a first step, periodic supercell DFT calculations are performed for the leads as well as the central region containing the molecule plus part of the leads. We use the Dacapo code (Bahn and Jacobsen 2002) which applies ultrasoft pseudopotentials (Vanderbilt 1990) for the ion cores. The KS eigenstates are expanded in plane waves with a cut off energy of 340 Ry and the PBE xc-functional (Perdew et al. 1996) is used. In the second step, the KS eigenstates are transformed into maximally localized, partially occupied WFs, $\left\{\phi_{n}(\mathbf{r})\right\}$, and the KS Hamiltonians of the central region and the leads are subsequently evaluated in terms of the WF basis. The eighteen maximally localized WFs obtained for the benzene molecule are shown in Fig. 14. The xc-potential is excluded from the Hamiltonian of the central region in order to avoid double counting when the $G W$ self-energy is added. The central region Hamiltonian reads

$$
\left[h_{C}\right]_{i j}=\left\langle\phi_{i}\left|-\frac{1}{2} \nabla^{2}+v_{p s}+v_{h}\right| \phi_{j}\right\rangle
$$

where $v_{p s}$ is the pseudopotential and $v_{h}$ is the Hartree potential. Notice that $v_{p s}$ and $v_{h}$ contain contributions from the ion cores and electron density of the leads as they are obtained from a supercell calculation with part of the leads included. 
Next, the Coulomb integrals are evaluated for the WFs of the central region,

$$
V_{i j, k l}=\iint \mathrm{d} \mathbf{r} \mathrm{d} \mathbf{r}^{\prime} \frac{\phi_{i}(\mathbf{r})^{*} \phi_{j}\left(\mathbf{r}^{\prime}\right)^{*} \phi_{k}(\mathbf{r}) \phi_{l}\left(\mathbf{r}^{\prime}\right)}{\left|\mathbf{r}-\mathbf{r}^{\prime}\right|}
$$

For the correlation part of the $G W$ self-energy, $\Sigma_{\text {corr }}=\Sigma_{G W}-\Sigma_{x}$, we use the effective interaction introduced in section 3.1, i.e. only Coulomb integrals of the form $V_{i j, i j}$ and $V_{i j, j i}$ are included. For the Hartree and exchange selfenergies, $\Sigma_{h}$ and $\Sigma_{x}$, which are easily evaluated from Eqs. $(58,59)$, we use all the Coulomb matrix elements. Notice, that we need $\Sigma_{h}$ even though the Hartree potential from electrons in $C$ is already contained in $v_{h}$. The reason is that the latter is the equilibrium Hartree potential of the DFT calculation, which might well differ from the Hartree potential of a nonequilibrium $G W$ calculation.

The retarded Green's function is evaluated from

$$
G^{r}=\left[\omega-h_{C}-\Sigma_{L}-\Sigma_{R}-\left(\Sigma_{h}^{r}[G]-\Sigma_{h}^{r}\left[g_{s}^{(\mathrm{eq})}\right]\right)-\Sigma_{x c}^{r}[G]\right]^{-1},
$$

where the frequency dependence has been omitted for notational simplicity. Several comments are in order. First, $\Sigma_{L}$ and $\Sigma_{R}$ are the lead self-energies of Eq. (41) (in the wide-band approximation $\Sigma_{L}$ and $\Sigma_{R}$ are diagonal and frequency independent). The term $\Delta v_{h} \equiv \Sigma_{h}^{r}[G]-\Sigma_{h}^{r}\left[g_{s}^{(\text {eq })}\right]$ is the change in Hartree potential relative to the equilibrium DFT value. This change is due to the applied bias and the replacement of $v_{x c}$ by $\Sigma_{x c}$. In this work $\Sigma_{x c}$ can be either the exchange or the $G W$ self-energy. Finally we notice that the bias dependence of the various quantities entering Eq. (90) has been suppressed for notational simplicity.

\subsection{Equilibrium spectrum of benzene}

In Fig. 15 we show the total density of states (DOS) of the isolated benzene molecule calculated using three different approximations: (i) DFT-PBE (ii) Hartree-Fock (iii) fully self-consistent $G W$. The DOS is given by

$$
D(\varepsilon)=-\frac{1}{\pi} \sum_{n=1}^{N_{w}} \operatorname{Im} G_{n n}^{r}(\varepsilon),
$$

where the sum runs over all WFs on the molecule, and the GF is obtained from Eq. (90) using a wide-band lead self-energy of $\Gamma=0.05$. We stress that our calculations include the full dynamic dependence of the $G W$ selfenergy as well as all off-diagonal elements. Thus no analytical extension is 
a)

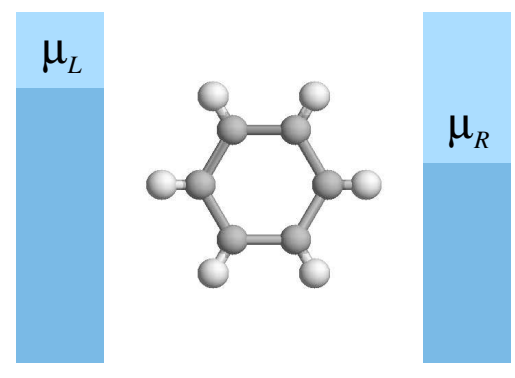

b)

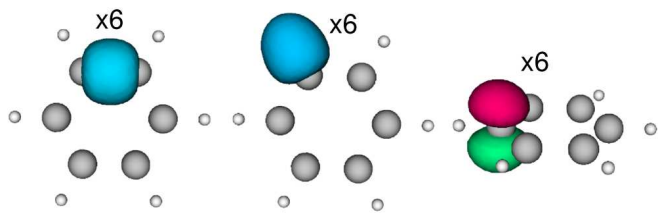

Figure 14: (a) Illustration of a benzene molecule coupled to featureless electrodes with different chemical potentials. (b) Iso-surfaces for the 18 partially occupied Wannier functions used as basis functions in the calculations. The WFs are linear combinations of Kohn-Sham eigenstates obtained from a DFT-PBE plane-wave calculation.

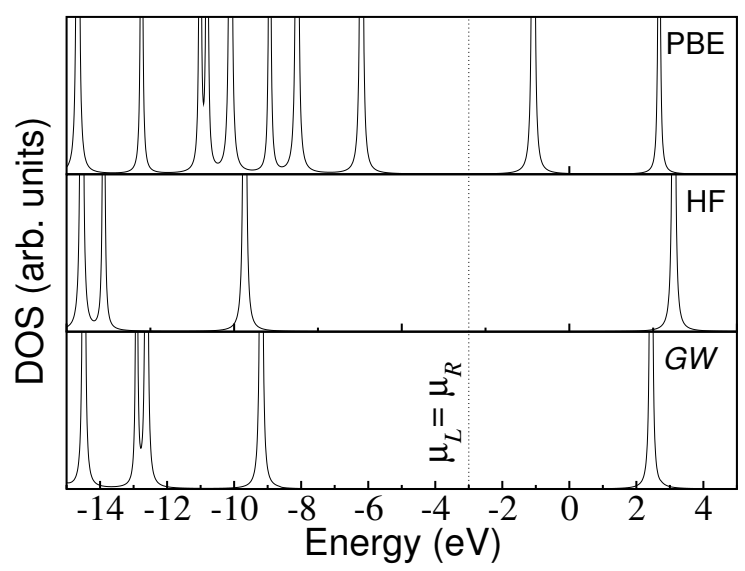

Figure 15: Density of states for a benzene molecule weakly coupled to featureless leads $(\Gamma=0.05)$. The common Fermi levels of the leads is indicated. Notice the characteristic opening of the band gap when going from DFT$\mathrm{PBE}$ to $\mathrm{HF}$, and the subsequent (slight) reduction when correlations are included at the $G W$ level. Reprinted with permission from Thygesen and Rubio, Phys. Rev. B 77, 115333 (2008). Copyright 2008 by the American Physical Society. 
performed, and we do not linearize the self-energy around the DFT eigenvalues to obtain an approximate quasi-particle equation as is done in standard $G W$ calculations.

The spectral peaks seen in Fig. 15 occurring above (below) the Fermi level correspond to electron addition (removal) energies. In particular, the HOMO level should coincide with the ionization potential of the isolated molecule, which in the case of benzene is $I_{\exp }=-9.2 \mathrm{eV}$ (NIST Chemistry WebBook). The PBE functional overestimates this value by $3 \mathrm{eV}$, giving $I_{\mathrm{PBE}}=-6.2 \mathrm{eV}$ in good agreement with previous calculations (Niehaus et al. 2005). The HF and $G W$ calculation yields $I_{\mathrm{HF}}=-9.7 \mathrm{eV}$ and $I_{G W}=-9.3 \mathrm{eV}$, respectively. Given the limited size of the Wannier basis, the precise values should not be taken too strict. However, the results demonstrate the general trend: KS theory with a local xc-functional underestimates the HOMO-LUMO gap significantly due to SI errors; HF overestimates the gap slightly; $G W$ reduces the HF gap slightly through the inclusion of dynamic screening.

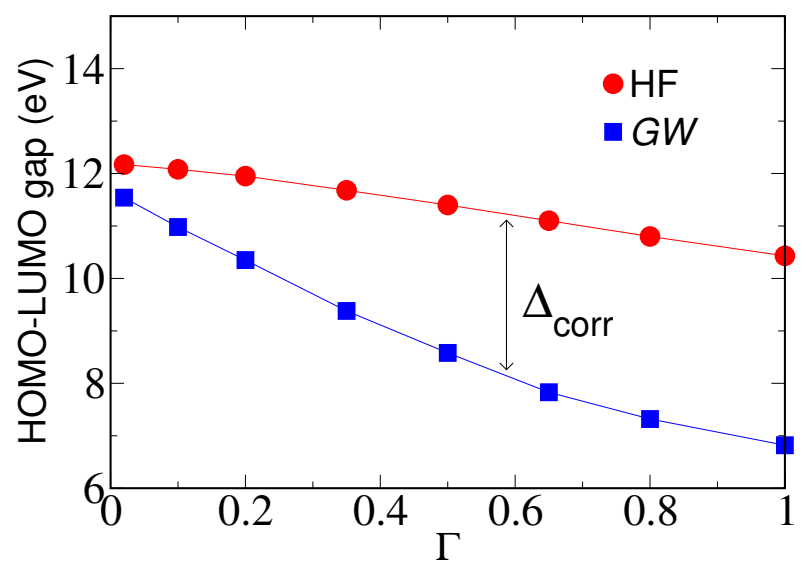

Figure 16: The HF and GW HOMO-LUMO gap of the benzene molecule as a function of the coupling strength $\Gamma$. The difference between the curves represents the reduction in the gap due to the correlation part of the $G W$ self-energy. This value increases with the coupling strength, as polarization of the molecule via dynamic charge transfer to the metal becomes possible (see section 5.2.3). Reprinted with permission from Thygesen and Rubio, Phys. Rev. B 77, 115333 (2008). Copyright 2008 by the American Physical Society.

In Fig. 16 we plot the size of the HOMO-LUMO gap as a function of 
the coupling strength $\Gamma$. The position of the levels has been defined as the first maximum in the DOS to the left and right of the Fermi level. Both the $\mathrm{HF}$ and $G W$ gaps decrease as $\Gamma$ is increased. This observation is consistent with the model calculations of section 5.2.3 where it was found that the gap reduction due to the correlation part of the $G W$ self-energy, $\Delta_{\text {corr }}$, can be understood as a virtual charge transfer between molecule and leads. The reduction of the HF gap as function of $\Gamma$ is a consequence of the redistribution of charge from the HOMO to the LUMO when the resonances broaden and their tails start to cross the Fermi level. This is completely analogue to the bias induced gap reduction discussed in section 5.3. These results for benzene show that the conclusions obtained from the two-level model apply to more realistic systems.

\subsection{Conductance of benzene}

We consider electron transport through the benzene junction under a symmetric bias, $\mu_{L / R}= \pm V / 2$, and a wide-band coupling strength of $\Gamma_{L}=\Gamma_{R}=$ $0.25 \mathrm{eV}$.

In Fig. 17 we compare the differential conductance, $\mathrm{d} I / \mathrm{d} V$, calculated from self-consistent DFT-PBE, HF, and $G W$, as well as non self-consistent $G_{0} W_{0}$ using either the DFT-PBE or HF Green's function as $G_{0}$. The $\mathrm{d} I / \mathrm{d} V$ has been obtained by numerical differentiation of the $I(V)$ curves calculated from Eq. (68). For the DFT calculation the finite-bias effects have been included at the Hartree level, i.e. changes in the xc-potential have been neglected. We notice that the $\mathrm{HF}$ and $G_{0} W_{0}\left[G_{\mathrm{HF}}\right]$ results are close to the self-consistent $G W$ result. These approximations all yield a nearly linear $I V$ with a conductance of $\sim 0.05 G_{0}$. In contrast the DFT and $G_{0} W_{0}\left[G_{\mathrm{DFT}}\right]$ yield significantly larger conductances which increase with the bias voltage. We note that the violation of charge conservation in the $G_{0} W_{0}$ calculations is not too large in the present case $(\Delta I / I<5 \%)$. This is in line with our general observation, e.g. from the Anderson model, that $\Delta I / I$ increases with $I$.

The trends in conductance can be understood by considering the (equilibrium) DOS of the junction shown in Fig. 18. As for the weakly-coupled (free) benzene molecule whose spectrum is shown in Fig. 15, the DFT HOMOLUMO gap is much smaller than the HF gap, and this explains the larger DFT conductance. The $G W$ gap falls in between the DFT and HF gaps, however, the magnitude of the DOS at $E_{F}$ is very similar in $G W$ and $\mathrm{HF}$ which is the reason for the similar conductances. It is interesting to notice that the HOMO-LUMO gap obtained in the $G_{0} W_{0}$ calculations resemble the gap obtained from $G_{0}$, and that the self-consistent $G W$ gap lies in between the $G_{0} W_{0}\left[G_{\mathrm{DFT}}\right]$ and $G_{0} W_{0}\left[G_{\mathrm{HF}}\right]$ gaps. 


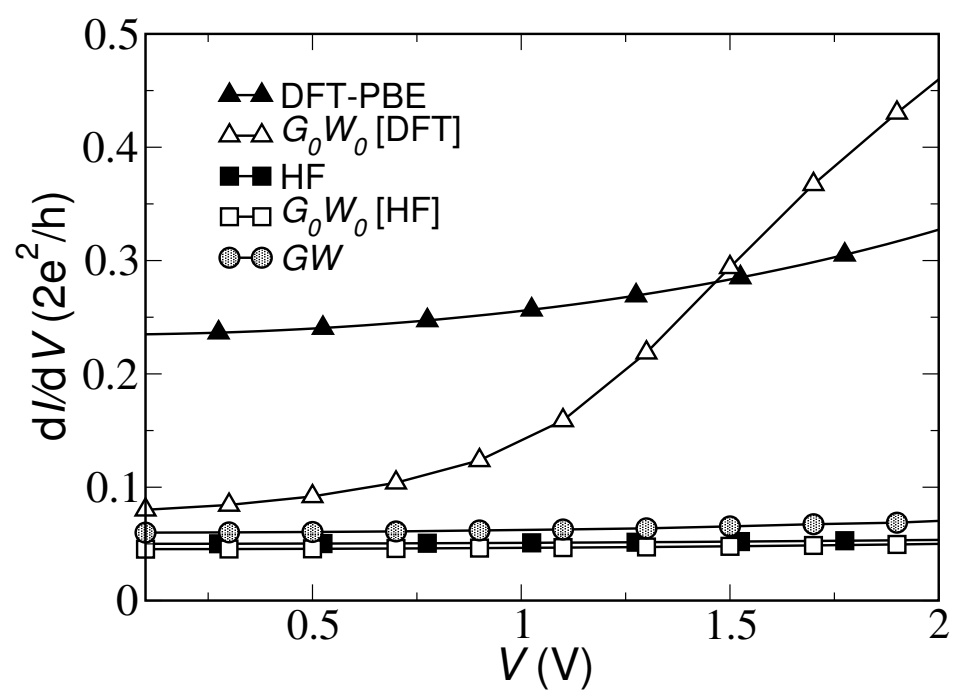

Figure 17: Differential conductance of the benzene junction for $\Gamma_{L}=\Gamma_{R}=$ $0.25 \mathrm{eV}$. Notice the strong $G_{0}$ dependence of the $G_{0} W_{0}$ result. Reprinted with permission from Thygesen and Rubio, Phys. Rev. B 77, 115333 (2008). Copyright 2008 by the American Physical Society.

The increase in the $G_{0} W_{0}\left[G_{\mathrm{DFT}}\right]$ conductance as a function of bias occurs because the LUMO of the $G_{0} W_{0}\left[G_{\mathrm{DFT}}\right]$ calculation moves downwards into the bias window and becomes partly filled as the voltage is raised. In a self-consistent calculation this would lead to an increase in Hartree potential which would in turn raise the energy of the level. The latter effect is missing in the perturbative $G_{0} W_{0}$ approach and this can lead to uncontrolled changes in the occupations as the present example shows.

Finally, we notice that the $G_{0} W_{0}\left[G_{\mathrm{DFT}}\right]$ DOS is significantly more broadened than both the $G_{0} W_{0}\left[G_{\mathrm{HF}}\right]$ and $G W$ DOS. The reason for this is that, as a direct consequence of the small HOMO-LUMO gap, DFT yields a the larger DOS close to $E_{F}$. The larger DOS in turn enhances the QP scattering and leads to shorter life-times of the QP in the $G_{0} W_{0}\left[G_{\mathrm{DFT}}\right]$ calculation. Since the QP life-time is inversely proportional to $\operatorname{Im} \Sigma_{G W}$ this explains the broadening of the spectrum. 


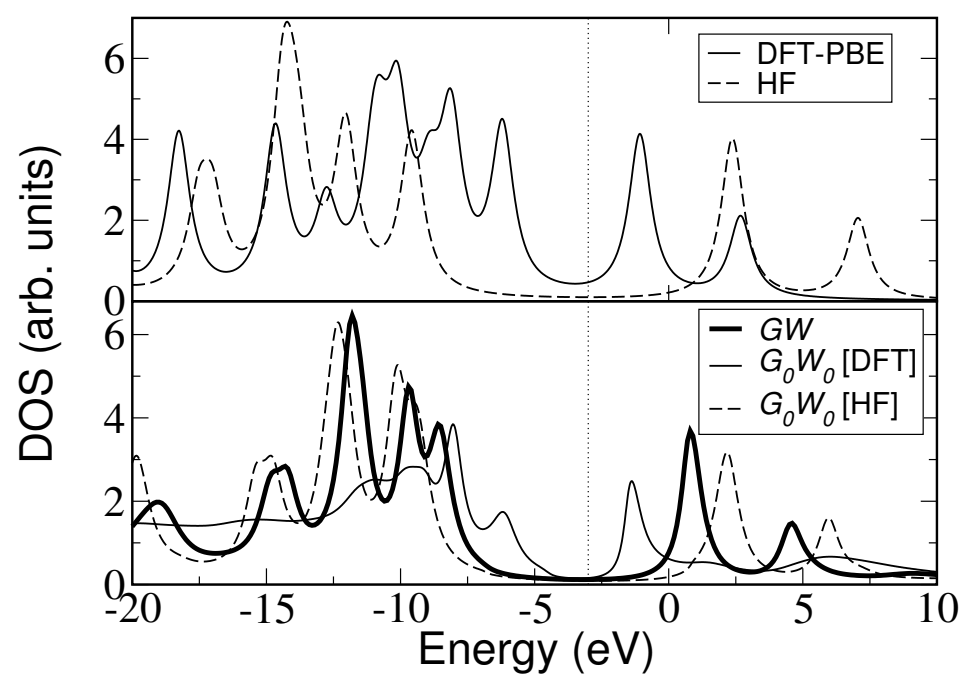

Figure 18: Equilibrium DOS for the benzene molecule coupled to wide-band leads with a coupling strength of $\Gamma_{L}=\Gamma_{R}=0.25 \mathrm{eV}$. Upper panel shows DFT-PBE and HF single-particle approximations while the lower panel shows the self-consistent $G W$ result as well as one-shot $G_{0} W_{0}$ results based on the DFT and HF Green's functions, respectively. Reprinted with permission from Thygesen and Rubio, Phys. Rev. B 77, 115333 (2008). Copyright 2008 by the American Physical Society.

\section{4 $\quad \mathrm{Pt}^{-\mathrm{H}_{2}}$-Pt junction}

We consider a molecular hydrogen bridge between infinite atomic Pt chains as shown in the inset of Fig. 19. Experimentally, the conductance of the hydrogen junction is found to be close to the conductance quantum, $G_{0}=2 e^{2} / h$ (Smit et al. 2002), and this value has been reproduced by DFT calculations (Thygesen and Jacobsen 2005). Below we present $G W$-transport results for a simplified model of this system (using infinite Pt chains as leads), and refer to Thygesen and Rubio 2007 for further details on the calculations.

In the upper panel of Fig. 19 we show the local density of states (LDOS) at one of the two $\mathrm{H}$ orbitals as calculated within DFT using the PBE xcfunctional, as well as self-consistent HF (in the central region). In DFT the $\mathrm{H}_{2}$ bonding state is a bound state at $-7.0 \mathrm{eV}$ relative to $E_{F}$, while the antibonding state lies at $0.4 \mathrm{eV}$ and is strongly broadened by coupling to the Pt. Moving from DFT to HF the bonding state is shifted down by $\sim 8 \mathrm{eV}$ 


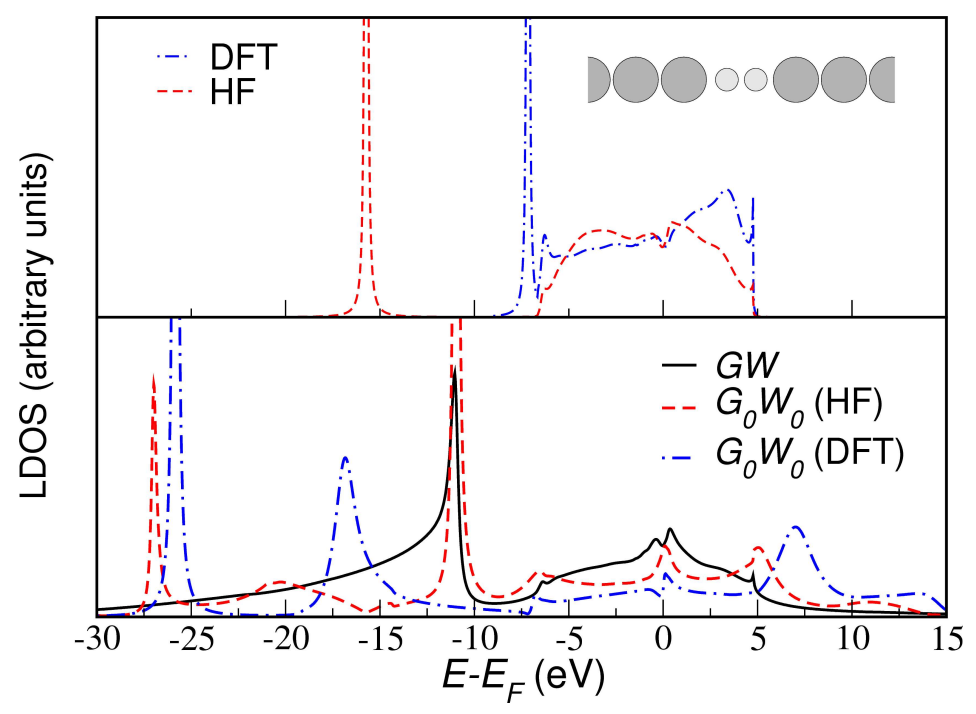

Figure 19: Local density of states at one of the $\mathrm{H}$ orbitals of the Pt-HH-Pt contact shown in the inset. Reprinted with permission from K. S. Thygesen and A. Rubio, J. Chem. Phys. 126, 091101 (2007). Copyright 2007, American Institute of Physics.

because for occupied states the exchange potential is more negative than the DFT xc-potential. The same effect tends to drive the half-filled anti-bonding state down but in this case the resulting increase in the Hartree potential (about $4 \mathrm{eV}$ ) stops it just below $E_{F}$.

In the lower panel of Fig. 19 we show the LDOS calculated in $G W$ as well as $G_{0} W_{0}$ starting from either DFT or HF, i.e. $G_{0}$ is either $G_{\mathrm{DFT}}$ or $G_{\mathrm{HF}}$. The large deviation between the two $G_{0} W_{0}$ results is not surprising given the large difference between $G_{\mathrm{DFT}}$ and $G_{\mathrm{HF}}$. A closer analysis of the origin of this deviation can be found elsewhere (Thygesen and Rubio 2007). We are, however, aware that part of this large difference could be due to the limited size of the basis. We also mention that the LDOS results of Fig. 19 can be largely reproduced by including only the second-order $G W$ diagram in the self-energy. Thus the higher-order RPA diagrams are less important in this case.

In Fig. 20 we show the self-consistently calculated $I V$ characteristics in DFT and $G W$. At low bias both schemes yield a conductance close to the experimental value of $1 G_{0}$. The DFT conductance is nearly constant over the bias range, and is in fact very similar to the HF result (not shown). In contrast the $G W$ conductance falls off at larger bias. This is due to enhancement of quasi-particle scattering at finite bias. The QP scattering 


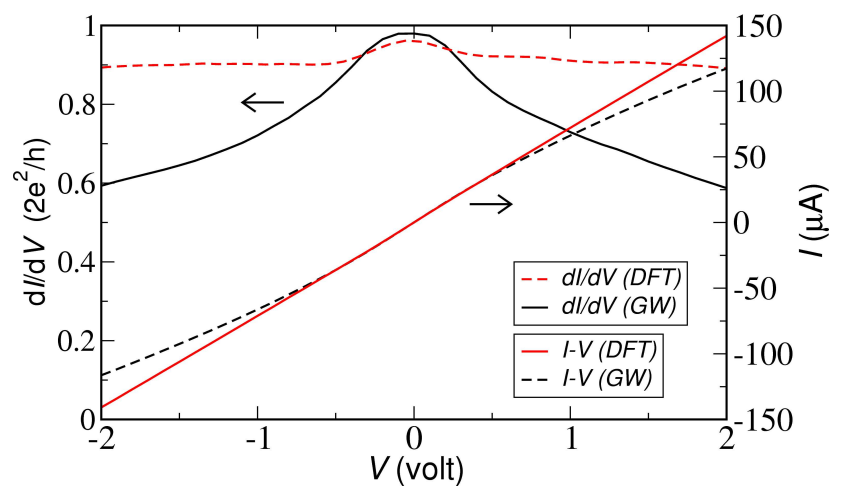

Figure 20: $I-V$ and $d I / d V$ for the hydrogen contact as calculated in DFT(PBE) and self-consistent $G W . V$ is the source-drain bias voltage. Reprinted with permission from K. S. Thygesen and A. Rubio, J. Chem. Phys. 126, 091101 (2007). Copyright 2007, American Institute of Physics.

reduces the life time of the QPs leading to broadening of the spectral peak associated with the anti-bonding state of the hydrogen molecule in agreement with the results for he two level model discussed in section 5.3. Since the correlation induced life-time of QP at the Fermi level, $\operatorname{Im} \Sigma_{\text {corr }}\left(E_{F}\right)$, vanishes identically in equilibrium, the finite-bias conductance suppression seen in figure 20 is a direct result of the non-equilibrium treatment of correlations.

\section{SUMMARY AND PERSPECTIVES}

The feasibility of using many-body perturbation theory in combination with the Keldysh Green's function formalism to address nonequilibrium quantum transport through an interacting region coupled to non-interacting leads has been demonstrated. The effect of electronic correlations was incorporated into the Green's function via the $G W$ self-energy, and the coupling to leads was treated exactly (to all orders in the hopping between leads and central region). The important connection between self-consistency in the $G W$ selfenergy and charge conservation was emphasized, and it was demonstrated that a non self-consistent treatment of the $G W$ self-energy (the $G_{0} W_{0}$ approach) violates the continuity equation and produce unphysical results at finite bias. This, together with the arbitrariness of the $G_{0} W_{0}$ approximation due to its $G_{0}$-dependence, speaks in favor of the self-consistent $G W$ approach to nonequilibrium transport.

The role of dynamic correlation effects in quantum transport was illustrated by applying the $G W$-transport scheme to a generic two-level model, 
a benzene molecule between featureless leads, and a hydrogen molecule between infinite $\mathrm{Pt}$ chains. It was shown that dynamic polarization of the leads as well as the molecule itself can lead to significant reduction of the molecule's HOMO-LUMO gap. The polarization effects were found to increase with the bias voltage where also quasiparticle scattering is strongly enhanced leading to broadening of the molecular resonances. Both effects can have a large impact on the calculated $I V$ curve, demonstrating the important role of dynamic correlation in quantum transport. This should always be kept in mind, when interpreting results of meanfield (DFT or Hartree-Fock) transport calculations - in particular under finite bias.

As mentioned in the introduction, the quantitative theoretical description of quantum transport in nano-scale structures from first principles is an extremely complex problem. Nevertheless, simulations methods with predictive power are required to advance the field further. It has been known for several years that the standard DFT-NEGF scheme fails to predict even the zero-bias conductance of certain classes of systems. This state of affairs makes it difficult, although not impossible, to link theory and experiments and thereby stimulate the development of nano-scale electronics.

As illustrated by the examples given in this chapter, reliable schemes for quantum transport should account for dynamic correlations effects in some way or another. The $G W$ method discussed here includes some correlation effects, but misses others, e.g. the side peaks in the spectral function of the Anderson model are not well reproduced (Thygesen and Rubio 2007). Methods developed for strongly correlated systems, such as density matrix renormalization group theory (Schmitteckert and Evers 2008), are limited to simple models due to their inexpedient scaling with system size. The effective single-particle scheme of TDDFT makes it an attractive alternative to many-body perturbation theory, in particular for dynamical transport phenomena (Stefanucci et al. 2007). However, the inclusion of correlation effects requires use of xc-potentials with memory which have so far proved difficult to construct.

Another important aspect of the problem is related to the coupling between electrons and nuclei. Despite the large difference in the general time scales of electronic and nuclear motions, electronic wavepackets quite often couple with the dynamics of nuclear motion (Frederiksen et al. 2004, Verdozzi et al. 2006). The proper incorporation of the electronic-nuclear interaction is crucial for describing a host of dynamical processes including Joule heating, electromigration, laser-induced electronic transport and electron transfer in molecular, biological, or electrochemical systems. Within the groundstate DFT framework, the computation of forces on the nuclei is trivial thanks to the Hellman-Feynman theorem. The situation is more complex out of 
equilibrium, and even more so in combination with a many-body description of the electrons, where the Hellman-Feynman theorem does not apply. However, the electron-ion dynamics must eventually be taken properly into account for a realistic description of a large class of molecular devices relevant for technological applications such as fast, integrated, optoelectronic nanodevices.

\section{ACKNOWLEDGMENTS}

KST acknowledges support from the Danish Center for Scientific Computing through grant No. HDW-1103-06 and The Lundbeck Foundation's Center for Atomic-scale Materials Design (CAMD). AR acknowledge funding by the Spanish MEC (FIS2007-65702-C02-01), "Grupos Consolidados UPV/EHU del Gobierno Vasco" (IT-319-07), and by the European Community through NoE Nanoquanta (NMP4-CT-2004-500198), e-I3 ETSF project (INFRA2007-1.2.2: Grant Agreement Number 211956) SANES(NMP4-CT-2006-017310), DNA-NANODEVICES (IST-2006-029192) and NANO-ERA Chemistry projects and the computer resources provided by the Barcelona Supercomputing Center, the Basque Country University UPV/EHU (SGIker Arina).

\section{APPENDIX}

Let $B\left(\tau, \tau^{\prime}\right)$ and $C\left(\tau, \tau^{\prime}\right)$ be two matrix valued functions on the Keldysh contour, and consider the commutator $A$ defined by

$$
A\left(\tau, \tau^{\prime}\right)=\int_{\mathcal{C}}\left[B\left(\tau, \tau_{1}\right) C\left(\tau_{1}, \tau^{\prime}\right)-C\left(\tau, \tau_{1}\right) B\left(\tau_{1}, \tau^{\prime}\right)\right] \mathrm{d} \tau_{1}
$$

where matrix multiplication is implied. Under steady state conditions where the real time components of $B$ and $C$ can be assumed to depend only on the time difference $t^{\prime}-t$, the following identity holds:

$$
\operatorname{Tr}\left[A^{<}(t, t)\right]=\int \frac{\mathrm{d} \omega}{2 \pi} \operatorname{Tr}\left[B^{<}(\omega) C^{>}(\omega)-B^{>}(\omega) C^{<}(\omega)\right] .
$$

To prove this relation we first use the Langreth rules to obtain

$$
\begin{aligned}
A^{<}\left(t, t^{\prime}\right) & =\int\left[B^{<}\left(t, t_{1}\right) C^{a}\left(t_{1}, t^{\prime}\right)+B^{r}\left(t, t_{1}\right) C^{<}\left(t_{1}, t^{\prime}\right)\right. \\
& \left.-C^{<}\left(t, t_{1}\right) B^{a}\left(t_{1}, t^{\prime}\right)-C^{r}\left(t, t_{1}\right) B^{<}\left(t_{1}, t^{\prime}\right)\right] \mathrm{d} t_{1} .
\end{aligned}
$$


Since all quantities on the right hand side depend only on the time difference we identify the integrals as convolutions which in turn become products when Fourier transformed. We thus have

$$
\begin{aligned}
A^{<}(t, t)= & \int \frac{\mathrm{d} \omega}{2 \pi} A^{<}(\omega) \\
= & \int \frac{\mathrm{d} \omega}{2 \pi}\left[B^{<}(\omega) C^{a}(\omega)+B^{r}(\omega) C^{<}(\omega)\right. \\
& \left.\quad-C^{<}(\omega) B^{a}(\omega)-C^{r}(\omega) B^{<}(\omega)\right] .
\end{aligned}
$$

Eq. (93) now follows from the cyclic property of the trace and the identity $G^{r}-G^{a}=G^{>}-G^{<}$. 


\section{REFERENCES}

Anderson, P.W. (1961) Phys. Rev. 12441.

Bahn, S. R. and Jacobsen, K. W. (2002) Comp. Sci. Eng.4 56.

Baym, G. (1962), Phys. Rev. 1271391.

Baym, G., and Kadanoff, L. P. (1962) Phys. Rev. 124287.

Bokes, P., Jung, J., Godby, R. W. (2007) Phys. Rev. B 76125433.

Brandbyge, M., Mozos, J. L., Ordej0n, P., Taylor, J., and Stokbro, K. (2002)

Phys. Rev. B 65165401.

Coleman, P. (1984) Phys. Rev. B 293035.

Costi, T. A., Hewson, A. C. and Zlatic V. (1994) J. Phys. Cond. Matt. 6 2519.

Cuniberti, G., Fagas, G., and Richter, K. (2005) Introducing molecular electronics, Springer.

Datta, S. (1995) Electronic Transport in Mesoscopic Systems, Cambridge University Press.

Delaney, P., Greer, J. C. (2004) Phys. Rev. Lett. 93036805.

Di Ventra, M., Todorov, T. N. (2004) J. Phys.:Condens. Matter 168025.

Dubois, M., Latil, S., Scifo, L., Grvin, B., and Rubio, A. (2006), J. Chem. Phys. 125 34708-9.

Frederiksen, T., Brandbyge, M., Lorente, N., and Jauho, A.-P. (2004) Phys. Rev. Lett. 93, 256601.

García-Suárez, V. M., Rocha, A. R., Bailey, S. W., Lambert, C. J., Sanvito, S., and Ferrer, J. (2005), Phys. Rev. Lett. 95256804.

Goldhaber, -G. D., Shtrikman, H., Mahalu, D., Abusch-Magder, D., Meirav, U. and Kastner, M. A. (1998) Nature 391156.

Haug, H. and Jauho, A.-P. (1998) Quantum Kinetics in Transport and Optics of Semiconductors, Springer.

Hershfield, S., Davies, J.H. and Wilkins J. W. (1991) Phys. Rev. Lett. 67 3720 .

Hettler, M. H., Wenzel, W., Wegewijs, M. R., Schoeller, H. (2003) Phys. Rev. Lett. 90076805.

Heurich, J., Cuevas, J. C., Wenzel, W. and Schon, G. (2002) Phys. Rev. Lett. 88256803.

Hybertsen, M. S. and Louie, S. G. (1986) Phys. Rev. B 345390.

Joachim, C., Gimzewski, J. K., Aviram, A. (2000) Nature 408, 541.

Johnson, P. D., and Hulbert, S. L. (1987), Phys. Rev. B 359427.

Kubatkin, S., Danilov, A., Hjort, M., Cornil, J., Bredas, J.-L., Stuhr-Hansen, N., Hedeård, P., and Bjørnholm, T. (2003), Nature 425, 698. 
Landauer, R. (1970), Phil. Mag. 21863.

Leeuwen, R. van, Dahlen, N.E., Stefanucci, G., Almbladh, C.O. and Barth, U. von (2006) Time-Dependent Density Functional Theory, Springer.

Meir, Y. and Wingreen, N. S. (1992), Phys. Rev. Lett. 682512.

Mowbray, D. J., Jones, G., and Thygesen, K. S. (2008), J. Chem. Phys. 128 111103.

Neaton, J. B., Hybertsen, M. S., and Louie, S. G. (2006), Phys. Rev. Lett. 97216405.

Nelson, W., Bokes, P., R. Patrick, and Godby, R.W. (2007) Phys. Rev. A 75032505 .

Niehaus, T. A., Rohlfing, M., Della, F. S., Di Carlo, A., Frauenheim, T. (2005) Phys. Rev. A 71022508.

NIST Chemistry WebBook, http://webbook.nist.gov/chemistry/

Nygard, J., Cobden, D.H., Lindelof, P.E. (2000) Nature 408 342-6.

Onida, G., Reining, L., and Rubio, A. (2002), Rev. Mod. Phys. 74601.

Perdew, J. P., Burke, K., and Ernzerhof, M. (1996), Phys. Rev. Lett. 77 3865.

Pulay, P. (1980) Chem. Phys. Lett. 73393.

Quek, S. Y., Venkataraman, L., Choi, H. J., Louie, S. G., Hybertsen, M. S., and Neaton, J. B. (2007), Nano Lett. 73477.

Reichert, J., Ochs, R., Beckman, D., Weber, H. B., Mayor, M. and Löhneysen, H. (2002) Phys. Rev. Lett. 88176804.

Repp, J., Meyer, G., Stojkovic, S. M., Gourdon, A., and Joachim, C. (2005), Phys. Rev. Lett. 94026803.

Smit, R. H. M.,Noat, Y., Untiedt, C., Lang, N. D., Hemert, M. C., and Ruitenbeek, J. M. (2002) Nature 419906.

Schmitteckert, P., Evers, F. (2008) Phys. Rev. Lett. 100, 086401.

Spataru, C. D, Benedict, L. X., and Louie, S. G. (2004), Phys. Rev. B 69 205204.

Stan, A., Dahlen, N. E., and Leeuwen, R. van (2006), Europhys. Lett. 76 298.

Stefanucci, G., Kurth, S., Gross, E. K. U., Rubio, A. (2007) Ed. J. Seminario $17247-284$.

Taylor, J., Guo, H., and Wang, J. (2001) Phys. Rev. B 63245407.

Thygesen, K. S. (2008), Phys. Rev. Lett. 100166804.

Thygesen, K. S. (2006) Phys. Rev. B 73035309.

Thygesen, K. S. and Jacobsen, K. W. (2005) Phys. Rev. Lett. 94036807.

Thygesen, K.S. and Rubio, A. (2007), J. Chem. Phys. 126091101.

Thygesen, K.S. and Rubio, A. (2008), Phys. Rev. B 359427.

Toher, C., Filippetti, A., Sanvito, S., and Burke, K. (2005), Phys. Rev. Lett. 95146402. 
Vanderbilt, D. (1990), Phys. Rev. B 417892.

Verdozzi, C., Godby, R. W., and Holloway, S. (1995), Phys. Rev. Lett. 74 2327.

Verdozzi, C., Stefanucci, G., Almbladh, C.-O. (2006) Phys. Rev. Lett. 97, 046603.

Wilson, K.G. (1975) Rev. Mod. Phys. 47773. 